\title{
Thermodynamical Frameworks for Higher Grade Material Theories with Internal Variables or Additional Degrees of Freedom
}

\author{
Christina Papenfuss ${ }^{1, \star}$ and Samuel Forest ${ }^{2}$ \\ 1 Technische Universität Berlin, Institut für Mechanik, Straße des 17. Juni 135, \\ 10623 Berlin, Germany \\ ${ }^{2}$ Ecole des Mines de Paris, CNRS, Centre des Matériaux, UMR 7633 BP 87, \\ 91003 Evry, France
}

*Corresponding author (c.papenfuss@gmx.de)

Communicated by W. Muschik, Berlin, Germany

\begin{abstract}
The objective of the present work is to compare several thermomechanical frameworks, taking into account the influence of strain gradient, internal variables, gradient of internal variables, and temperature gradient on the constitutive behavior of materials. In particular, the restrictions by the second law of thermodynamics are derived. The method of exploitation consists of two steps: an application of the well-known method by Liu and a new method of exploiting the residual inequality. The first example introduces an enlarged set of variables for the constitutive functions including in particular the strain gradient, an internal variable, its gradient, and the temperature gradient. In the second example, the power of internal forces is enriched to incorporate generalized stress measures. In the third example, the classical thermomechanical setting is complemented by a balance-type differential equation for an additional variable. Finally, material theories of grade $n$ are envisaged. It is shown that the free energy density may depend on gradients only in the case that an additional balance equation is introduced. We also demonstrate that for isotropic materials the second law of thermodynamics implies for a large class of state spaces that the entropy flux equals the heat flux divided by temperature.
\end{abstract}




\section{Introduction}

\subsection{Scope of this work}

A precise description of the thermoelastoviscoplastic behavior of materials based on phenomenological constitutive equations requires the introduction of internal variables [1-4]. An example of such an internal variable is the alignment tensor in liquid crystals [5-7], and it is known that in this case the transport of orientational order gives an extra contribution to the entropy flux [8]. For an overview over the field and for different applications of internal variables, see for instance [9-16].

It is also possible within the continuum mechanical framework to account for the size-dependent material behavior observed in many physical situations (like grain size effects in polycrystals or particle size effects in composites) by introducing strain gradients or gradients of internal variables into the constitutive modelling [17-20].

When the constitutive functions like stress and free energy density are assumed to depend on additional variables with respect to the classical framework, such as strain gradients and/or gradients of internal variables, the thermodynamical setting of the theory must be reconsidered. In particular, the restrictions on such dependences induced by the second law of thermodynamics must be examined. Three main trends can be distinguished in the literature to tackle this problem:

- In many cases, the thermomechanical consistency of proposed strain gradient models is not checked systematically. In particular, the balance equations (momentum and energy) are assumed to keep their classical form so that the boundary value problem remains unaffected. This is the case for instance for the strain gradient plasticity models in [21-23]. Some limitations of such models are reviewed in [24].

- The incorporation of higher order gradient effects is made possible in some theories by enriching the power of internal forces or by adding contributions to the energy balance. The mechanical power is enriched in the second gradient of displacement theories [25-27] by introducing higher order generalized stress tensors. Such a generalized contribution is added at the level of the energy balance in [28]. The case of the gradient of damage is tackled in [29] by extending the power of internal forces through the product of the gradient of damage rate by a generalized stress measure. Mindlin's theory of second gradient media is extended to thermoelasticity in $[30,31]$ by introducing the gradient of temperature into the thermomechanical setting. Strain gradient plasticity models are proposed in $[32,33]$. These extensions 
lead to additional balance equations and additional boundary conditions to be taken into account.

- The introduction of an extra entropy flux $\underline{k}$ has been considered as an alternative generalization of the classical theory in $[34,10]$ to incorporate internal variables and their gradients. An extra entropy flux in the case of materials with additional degrees of freedom has been proposed in [9].

In contrast to these approaches, in [35] the problem of thermoviscous fluids is treated with heat flux and stress tensor as independent variables in the spirit of extended thermodynamics. Assuming that there is no influence of gradients, the authors find the classical expression for the entropy flux being heat flux over temperature. Including gradients into the set of variables, they find an extra entropy flux, i.e., a modified relation between entropy flux and heat flux.

The objective of the present work is to compare several thermomechanical frameworks taking the influence of strain gradient, gradient of internal variables, and temperature gradient on the constitutive behavior of materials into account. In particular, the restrictions on such constitutive dependences implied by the exploitation of the second law of thermodynamics are derived systematically and compared for the different frameworks. The method of exploiting the second law consists of two steps: The first step is an application of the well-known methods of Liu [36], or equivalently of the method of Coleman and Noll [37]. The second step is a new method of exploiting the residual inequality.

Section 2 considers the introduction of an enlarged state space including in particular the strain gradient, an internal variable, its gradient, and the temperature gradient, without considering any additional differential equation. The restrictions on the constitutive functional dependences are derived. In Section 3, the power of internal forces is enriched to incorporate generalized stress measures. In Section 4, the classical thermomechanical setting is complemented by a balance equation for the internal degree of freedom instead of the usual evolution equation. Finally, material theories of grade $n$ are envisaged in Section 5.

In this work, we reserve the term "internal variable" for a quantity which is neither observable nor controllable. The example of molecule or lattice rotation in liquid or solid crystal shows that a quantity can be observable (by Xray analysis for example) but not easily controllable because it is experimentally difficult to monitor it. The question then arises whether it should be treated as an internal variable or as an actual degree of freedom. When the gradient of such a variable plays a role in the material theory, we show especially in Section 3 that the variable can be treated as an additional degree of freedom. 
The analysis is confined to the case of solid bodies within the context of small perturbations, although straightforward extensions of most results are possible in the context of finite deformations or in the case of fluids. Accordingly, the privileged constitutive function in this work is the free energy density $\Psi$.

The notation used throughout this work is the following: scalar quantities, vectors, second- and third-rank tensors are denoted respectively, by $a, \underline{a}, \underset{\sim}{\boldsymbol{a}}$, a. Simple, double, and triple contraction read.,$:, \vdots \nabla$ is the nabla operator. The symmetric strain tensor is denoted by $\varepsilon$ within the context of small deformations. $\dot{a}$ is the time derivative of $a$.

\subsection{Method of exploiting the dissipation inequality}

According to the second law of thermodynamics, all processes are connected with a non-negative entropy production $\sigma \geq 0$. In principle, this could be guaranteed either by "ruling out" the (mathematical) solutions of the balance equations, which contradict this dissipation inequality, or by restrictions on constitutive functions, such that there exist only solutions of the balance equations together with constitutive equations in agreement with the dissipation inequality. With a very reasonable amendment to the second law, it can be shown that the second possibility is the case [38]: the second law of thermodynamics imposes restrictions on constitutive functions. The most general form of these restrictions is derived by the method of Liu [36] after having chosen the state space (the set of variables the constitutive functions depend upon). Let us give here a short sketch of the method:

The balance equations, after the chain rule is applied to the constitutive functions, form a system of equations linear in the so-called higher derivatives, i.e., the derivatives are not included in the state space but one order higher. These higher derivatives will be put together in a row $\boldsymbol{y}$. Then the system of balance equations and the dissipation inequality can be written symbolically as:

$$
\begin{aligned}
& \mathrm{A} \cdot \boldsymbol{y}=\boldsymbol{C}, \\
& \boldsymbol{B} \cdot \boldsymbol{y} \geq \mathrm{D},
\end{aligned}
$$

with a matrix $\boldsymbol{A}$, rows $\boldsymbol{B}$ and $\boldsymbol{C}$, and a scalar function D. All these quantities are functions of the state space variables.

LIU's proposition [39, 36, 40]: Constitutive equations satisfy the relations

$$
\mathbf{B}(Z)=\lambda(Z) \cdot \mathbf{A}(Z),
$$




$$
\lambda(Z) \cdot \mathbf{C}(Z) \geq \mathrm{D}(Z)
$$

with state space functions $\lambda(Z)$. The entropy production density

$$
\sigma:=\lambda \cdot \mathbf{C}-\mathrm{D} \geq 0
$$

is independent of the process direction, i.e., it depends only on the state space elements and not on higher derivatives.

The set of equations (3) will be denoted as "LIU equations", and Eq. (4) is the residual inequality. The LIU equations are as many equations as there are higher derivatives (elements in the row $y$ ). These are more equations than there are unknown factors $\lambda$, which is the same number as the number of balance equations. The equations remaining after eliminating the unknowns $\lambda$ from the LIU equations are the restrictions on constitutive functions. After eliminating the multipliers $\lambda$ from the residual inequality, an expression for the entropy production is obtained. In the examples, it turns out that this expression is of the form of a sum of products where one factor can be denoted as a thermodynamic force and the other one as a thermodynamic flux, i.e., it is of the form

$$
\sigma=\sum_{i} v_{i} f_{i}\left(v_{j}\right) \geq 0
$$

One can show that for continuous functions $f_{i}\left(v_{j}\right)$ it follows [41] that $f_{i}$ is a homogeneous function,

$$
v_{i}=0 \Rightarrow f_{i}=0 \text {. }
$$

This fact as well as the LIU equations will be exploited in the following.

Another way of exploiting the inequality of the second law of thermodynamics is the Coleman-Noll procedure [42]. Both methods have been used for the results presented in this work and can be shown to lead to the same conclusions under conditions stated in Section 3.

\section{Application to a gradient theory with an internal variable}

A higher order theory involving the first and second gradients of the displacement field is envisaged in this section using the method proposed in [25]. The influence of an internal variable $\alpha$ and of its first gradient is also taken into account. A full thermomechanical framework is the aim of this part. 


\subsection{Balance equations and equation of motion for the internal variable}

The material is supposed to be non-micropolar, i.e., there is no internal angular momentum and the balance of angular momentum is not independent of the balance of momentum. The remaining balance equations are:

\section{Balance of mass:}

$$
\dot{\varrho}+\varrho \nabla \cdot \underline{v}=0,
$$

with mass density $\varrho$ and material velocity $\underline{v}$.

\section{Balance of momentum:}

$$
\varrho \underline{\dot{v}}-\nabla \cdot(\boldsymbol{\sigma}-\nabla \cdot \underline{\boldsymbol{S}})-\varrho \underline{f}=0 .
$$

$f$ is the specific density of volume forces, $\sigma$ denotes the Cauchy stress tensor,

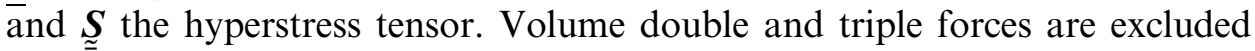
here for the sake of simplicity [26]. The special case of $\underset{\underline{S}}{\boldsymbol{S}}=0$ is discussed at the end of this section.

\section{Balance of internal energy:}

$$
\varrho \dot{e}+\nabla \cdot \underline{q}-\boldsymbol{\sigma}: \dot{\boldsymbol{\varepsilon}}-\underline{\underline{S}}: \nabla \dot{\dot{\varepsilon}}=0 .
$$

Here $e$ is the internal energy density, $q$ is the heat flux density, and the remaining terms are the (internal) power of stress and hyperstress. It is supposed that there is no radiation absorption.

We are dealing with solids, and we will restrict ourselves to small deformations. We introduce an additional internal variable $\alpha$ in order to model more complex material behavior, like for instance in the case of plasticity. The internal variable could be a scalar or a tensor of arbitrary order. For simplicity we introduce here a scalar internal variable, but the exploitation of the dissipation inequality works analogously with a tensor variable of arbitrary order.

For the internal variable we suppose an equation of motion of the form of a relaxation equation:

$$
\frac{d \alpha}{d t}=\pi_{\alpha}
$$

with a production $\pi_{\alpha}$ which is determined by internal dynamics. It is a constitutive quantity, depending on the whole set of state space variables, including the internal variable itself. 
The stress tensor $\boldsymbol{\sigma}$, the hyperstress $\underset{\underline{S}}{\boldsymbol{S}}$, the specific internal energy density $e$, and the heat flux $q$ are constitutive quantities. They depend on a set of variables, denoted as state space $\mathscr{Z}$ in a material-dependent manner. General conclusions concerning these constitutive functions, valid for any material, can be drawn from the second law of thermodynamics.

The second law of thermodynamics is expressed by the dissipation inequality:

$$
\sigma=\varrho \dot{\eta}+\nabla \cdot \underline{\Phi} \geq 0
$$

with specific entropy density $\eta$, entropy flux $\Phi$, and entropy production $\sigma$. The dissipation inequality is exploited according to Liu (see Section 2.2) together with the balances of mass, momentum, and internal energy, as well as the relaxation equation for the internal variable. The corresponding multipliers are denoted by $\Lambda^{\varrho}, \underline{\Lambda^{v}}, \Lambda^{e}$, and $\Lambda^{\alpha}$. These are functions of the state space variables.

\subsection{Exploitation of the second law according to Liu}

The second law of thermodynamics is fulfilled by any thermodynamic process, if and only if the following inequality is fulfilled:

$$
\begin{aligned}
\varrho \dot{\eta}+ & \nabla \cdot \underline{\Phi}+\Lambda^{\varrho}(\text { Balance of mass })+\underline{\Lambda^{v}} \cdot(\text { Balance of momentum }) \\
& +\Lambda^{e}(\text { Balance of internal energy }) \\
& +\Lambda^{\alpha}(\text { Relaxation of internal variable }) \geq 0 .
\end{aligned}
$$

The implications of this inequality on constitutive functions are exploited in the following.

We assume that for material behavior strain and strain gradient, temperature and temperature gradient, and the internal variable $\alpha$ together with its gradient are relevant. They are included in the domain of constitutive functions $\mathscr{Z}$. In addition, the possibility of first-order time derivatives of these variables being relevant for the material behavior is taken into consideration:

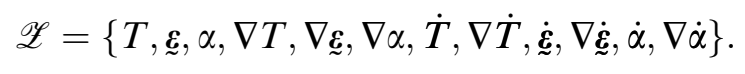

Constitutive quantities depend on position and time through the space and time dependence of the field quantities in the state space $\mathscr{Z}$. The time and space derivatives of all constitutive functions are carried out according to the chain rule, for example: 


$$
\begin{aligned}
\dot{e}= & \frac{\partial e}{\partial T} \frac{d T}{d t}+\frac{\partial e}{\partial \nabla T} \cdot \frac{d \nabla T}{d t}+\frac{\partial e}{\partial \boldsymbol{\varepsilon}}: \frac{d \underline{\varepsilon}}{d t} \\
& +\frac{\partial e}{\partial \nabla \boldsymbol{\varepsilon}}: \frac{d \nabla \boldsymbol{\varepsilon}}{d t}+\frac{\partial e}{\partial \alpha} \frac{d \alpha}{d t}+\frac{\partial e}{\partial \nabla \alpha} \cdot \frac{d \nabla \alpha}{d t} \\
& +\frac{\partial e}{\partial \dot{T}} \frac{d^{2} T}{d t^{2}}+\frac{\partial e}{\partial \nabla \dot{T}} \cdot \nabla \frac{d^{2} T}{d t^{2}}+\frac{\partial e}{\partial \dot{\boldsymbol{\varepsilon}}}: \frac{d^{2} \boldsymbol{\varepsilon}}{d t^{2}} \\
& +\frac{\partial e}{\partial \nabla \dot{\boldsymbol{\varepsilon}}} \vdots \nabla \frac{d^{2} \boldsymbol{\varepsilon}}{d t^{2}}+\frac{\partial e}{\partial \dot{\alpha}} \frac{d^{2} \alpha}{d t^{2}}+\frac{\partial e}{\partial \nabla \dot{\alpha}} \cdot \nabla \frac{d^{2} \alpha}{d t^{2}}
\end{aligned}
$$

Application of the chain rule to all constitutive functions in the inequality (13) leads to an expression linear in the so-called higher derivatives not included in the state space. These are in our case:

$$
\ddot{T}, \nabla \ddot{T}, \ddot{\boldsymbol{\varepsilon}}, \nabla \ddot{\boldsymbol{\varepsilon}}, \ddot{\alpha}, \nabla \ddot{\alpha}, \nabla \nabla T, \nabla \nabla \underline{\varepsilon}, \nabla \nabla \alpha, \nabla \nabla \dot{T}, \nabla \nabla \dot{\boldsymbol{\varepsilon}}, \nabla \nabla \dot{\alpha}, \underline{\underline{v}} .
$$

Corresponding to each higher derivative, there is an equation restricting constitutive functions. From this set of equations, the quantities $\Lambda^{\alpha}, \underline{\Lambda^{v}}$, and $\Lambda^{e}$ are eliminated. The results are most conveniently written in terms of the specific free energy density $\Psi:=e-T \eta$ and the extra entropy flux $\underline{k}=$ $\underline{\mathbf{\Phi}}-\frac{\underline{\underline{q}}}{T}$. The multiplier $\Lambda^{\varrho}$ cannot be calculated, but the balance of mass plays simply the role of a constraint,

$$
\dot{\varrho}=-\varrho \text { trace } \dot{\varepsilon} \text {. }
$$

The corresponding multiplier plays neither a role in the restrictions on constitutive functions, nor in the expression for the entropy production and may remain undetermined. The same holds for the multiplier $\Lambda^{\alpha}$, because the relaxation equation (11) is a relation between state space variables.

It is found that

$$
\Lambda^{e}=-\frac{\partial \eta}{\partial e}
$$

and $\Lambda^{e}$ is set equal to the inverse temperature

$$
\Lambda^{e}=\frac{1}{T}
$$

This is in agreement with thermostatics, and an argument in favor of this identification in non-equilibrium can be found in [43]. 
From the equation corresponding to the higher derivative $\underline{\dot{v}}$, we calculate:

$$
\underline{\Lambda^{v}}=\underline{0},
$$

which is taken into account in the following.

The remaining restrictions on constitutive functions from the second law are

$$
\begin{aligned}
& \frac{\partial \Psi}{\partial z_{i}}=0 \quad z_{i} \in\{\dot{T}, \nabla \dot{T}, \dot{\boldsymbol{\varepsilon}}, \nabla \dot{\boldsymbol{\varepsilon}}, \dot{\alpha}, \nabla \dot{\alpha}\} \\
& \frac{\partial \underline{\underline{k}}}{\partial u_{i}}=0 \quad u_{i} \in\{\nabla T, \nabla \underline{\boldsymbol{\varepsilon}}, \nabla \alpha, \nabla \dot{T}, \nabla \dot{\boldsymbol{\varepsilon}}, \nabla \dot{\alpha}\} .
\end{aligned}
$$

In summary, the exploitation of the second law with the balance equations as constraints has restricted the dependence of the extra entropy flux and of the free energy density to the following variables:

$$
\underline{k}(T, \dot{T}, \underline{\varepsilon}, \dot{\varepsilon}, \alpha, \dot{\alpha}) .
$$

The extra entropy flux does not depend on the highest gradients in the state space. The dependence of the free energy density is then

$$
\Psi(T, \nabla T, \boldsymbol{\varepsilon}, \nabla \boldsymbol{\varepsilon}, \alpha, \nabla \alpha) .
$$

The free energy density does not depend on any time derivative.

In addition to the above restrictions on constitutive functions, the method of Liu results in an expression for the entropy production, which is a function of the state space variables:

$$
\begin{aligned}
& \sigma=\left(-\frac{\varrho}{T} \frac{\partial \Psi}{\partial T}-\varrho \frac{e}{T^{2}}\right) \dot{T}-\frac{\varrho}{T} \frac{\partial \Psi}{\partial \nabla T} \cdot \nabla \dot{T} \\
& \left(-\frac{\varrho}{T} \frac{\partial \Psi}{\partial \boldsymbol{\Psi}}+\frac{1}{T} \boldsymbol{\sigma}\right): \dot{\boldsymbol{\varepsilon}}+\left(-\frac{\varrho}{T} \frac{\partial \Psi}{\partial \nabla \underline{\boldsymbol{\varepsilon}}}+\frac{\partial \underline{\underline{k}}}{\partial \dot{\boldsymbol{\varepsilon}}}+\frac{1}{T} \underset{\boldsymbol{S}}{\underline{\boldsymbol{S}}}\right) \vdots \nabla \dot{\boldsymbol{\varepsilon}} \\
& -\frac{\varrho}{T} \frac{\partial \Psi}{\partial \alpha} \dot{\alpha}+\left(-\frac{\varrho}{T} \frac{\partial \Psi}{\partial \nabla \alpha}+\frac{\partial \underline{k}}{\partial \dot{\alpha}}\right) \cdot \nabla \dot{\alpha} \\
& \left(\frac{\partial \underline{k}}{\partial T}+\frac{1}{T^{2}} \underline{q}\right) \cdot \nabla T+\frac{\partial \underline{k}}{\partial \underline{\varepsilon}}: \nabla \underline{\varepsilon}+\frac{\partial \underline{k}}{\partial \alpha} \cdot \nabla \alpha \geq 0 .
\end{aligned}
$$

This inequality is exploited now further making use of Eq. (7). 


\subsection{Exploitation of the residual inequality}

In order to make it more clearly readable for the following exploitation, we write the entropy production in the form of a formal scalar product between rows, as suggested in Section 1.2. It is understood that in the lines involving vectors or tensors the scalar product (contraction over all pairs of indices in components) has to be taken:

$$
\sigma=\left(\begin{array}{c}
-\frac{\varrho}{T} \frac{\partial \Psi}{\partial T}-\varrho \frac{e}{T^{2}} \\
-\frac{\varrho}{T} \frac{\partial \Psi}{\partial T} \\
-\frac{\varrho}{T} \frac{\partial \Psi}{\partial \underline{\xi}}+\frac{1}{T} \boldsymbol{\sigma} \\
-\frac{\varrho}{T} \frac{\partial \Psi}{\partial \nabla \underline{\varepsilon}}+\frac{\partial \underline{k}}{\partial \dot{\dot{\varepsilon}}}+\frac{1}{T} \underset{\boldsymbol{S}}{-} \\
-\frac{\varrho}{T} \frac{\partial \Psi}{\partial \alpha} \\
-\frac{\varrho}{T} \frac{\partial \Psi}{\partial \alpha}+\frac{\partial \underline{k}}{\partial \dot{\alpha}} \\
\frac{\partial \underline{k}}{\partial T}+\frac{1}{T^{2}} \underline{q} \\
\frac{\partial \underline{k}}{\partial \underline{\varepsilon}} \\
\frac{\partial \underline{k}}{\partial \alpha}
\end{array}\right) \cdot\left(\begin{array}{c}
\dot{T} \\
\nabla \dot{T} \\
\dot{\boldsymbol{\varepsilon}} \\
\nabla \dot{\boldsymbol{\varepsilon}} \\
\dot{\alpha} \\
\nabla \dot{\alpha} \\
\nabla T \\
\nabla \dot{\varepsilon} \\
\nabla \alpha
\end{array}\right) .
$$

Equation (26) clearly shows that the entropy production is a sum of products of state space variables and derivatives of constitutive functions. Under the presupposition that the dependence of the constitutive functions on the state space variables is continuous, it follows according to Eq. (7) that each constitutive quantity in the row at the left-hand side is a homogeneous function of the state space variable in the row at the right-hand side in the same line. This fact is exploited now, beginning in the last line of Eq. (26):

$$
\frac{\partial \underline{k}}{\partial \alpha}=f_{1}(\mathscr{Z}) \nabla \alpha
$$

where $f_{1}(\mathscr{Z})$ is up to now an arbitrary function of the state space variables. On the other hand, $\underline{k}$ does not depend on $\nabla \alpha$ as a consequence of the dissipation inequality [see Eq. (23)] and therefore, the left-hand side of Eq. (27), $\frac{\partial \underline{k}}{\partial \alpha}$, is also independent of $\nabla \alpha$. Consequently, the only possibility to fulfill Eq. (27) is:

$$
f_{1}=0 \Rightarrow \frac{\partial \underline{k}}{\partial \alpha}=0
$$

The extra entropy flux does not depend on the internal variable. 
The exploitation of the entropy production proceeds with the line above in Eq. (26). This shows analogously that $\underline{k}$ does not depend on strain:

$$
\frac{\partial \underline{k}}{\partial \underline{\varepsilon}}=f_{2}(\mathscr{Z}) \nabla \underline{\xi}
$$

where $f_{2}(\mathscr{Z})$ is up to now an arbitrary function of the state space variables. On the other hand, $\underline{k}$ does not depend on $\nabla \varepsilon$ according to the Liu procedure [see Eq. (23)] and therefore, the left-hand side of Eq. (29), $\frac{\partial \underline{k}}{\partial \underline{\varepsilon}}$, is also indepen-
dent of $\nabla \varepsilon$, and the only way to fulfill Eq. (29) is

$$
f_{2}(\mathscr{Z})=0 \Rightarrow \frac{\partial \underline{k}}{\partial \underline{\varepsilon}}=0 .
$$

The exploitation of the next line in the entropy production does not restrict the extra entropy flux $k$, because of the unknown constitutive function heat flux $q$ in the corresponding equation. Concerning the dependence of the extra entropy flux $\underline{k}$ on temperature, we cannot draw any conclusion from the entropy production.

The results on the extra entropy flux and the free energy density derived up to here can be summarized as:

$$
\underline{k}(T, \dot{T}, \dot{\boldsymbol{\varepsilon}}, \dot{\alpha})
$$

Apart from temperature, the extra entropy flux depends only on time derivatives, whereas

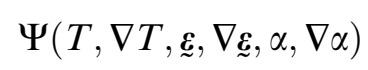

does not depend on any time derivative, but on gradients.

Further conclusions are drawn from the exploitation of the remaining lines of the entropy production. The next line in Eq. (26) results in

$$
-\frac{\varrho}{T} \frac{\partial \Psi}{\nabla \alpha}+\frac{\partial \underline{k}}{\partial \dot{\alpha}}=f_{3}(\mathscr{Z}) \nabla \dot{\alpha}
$$

with an arbitrary state space function $f_{3}(\mathscr{Z})$.

$\Psi$ and $\underline{k}$ both do not depend on $\nabla \dot{\alpha}$, and therefore the left-hand side of Eq. (33) is independent of $\nabla \dot{\alpha}$. Therefore, Eq. (33) can hold only if $f_{3}=0$. It follows 


$$
\frac{\varrho}{T} \frac{\partial \Psi}{\partial \nabla \alpha}=\frac{\partial \underline{k}}{\partial \dot{\alpha}}
$$

The left-hand side of Eq. (34) depends on $(T, \nabla T, \varepsilon, \nabla \varepsilon, \alpha, \nabla \alpha)$ [see Eq. (24)], whereas the right-hand side depends on $(T, \dot{\varepsilon}, \dot{\alpha})$. The only common variable is the temperature $T$, and we conclude that both sides of Eq. (34) can solely be functions of temperature. On the other hand, $\frac{\varrho}{T} \frac{\partial \Psi}{\partial \nabla \alpha}$ as well as $\frac{\partial \underline{k}}{\partial \dot{\alpha}}$ are vectors. An isotropic vector function cannot be represented by a scalar variable $T$. It follows:

$$
\frac{\varrho}{T} \frac{\partial \Psi}{\partial \nabla \alpha}=0, \quad \frac{\partial \underline{k}}{\partial \dot{\alpha}}=0
$$

Analogously, the line above in Eq. (26) is exploited and leads to the conclusion that $\frac{\varrho}{T} \frac{\partial \Psi}{\partial \alpha}$ is a homogeneous function of $\dot{\alpha}$ on one hand, but on the other hand $\Psi$ does not depend on $\dot{\alpha}$. It follows

$$
\frac{\varrho}{T} \frac{\partial \Psi}{\partial \alpha}=0
$$

i.e., we have shown that the free energy density does not depend on the internal variable $\alpha$. In the context of the elastic-viscoplastic behavior of materials, a dependence of the free energy on the internal variable $\alpha$ is required. This is usually done by postulating the existence of a convex dissipation potential $\Omega$ being a function of the thermodynamical force $a=\partial \Psi / \partial \alpha$. The evolution equation for $\dot{\alpha}$ is then given by the partial derivative $\partial \Omega / \partial a$. This framework proposed in $[1-3]$ is not addressed in the present work.

The exploitation of the next two lines in the entropy production equation (26) does not lead to restrictions on the free energy density and on the extra entropy flux due to the additional undetermined constitutive functions stress tensor $\underset{\sigma}{ }$ and hyperstress $\underset{\underline{S}}{\boldsymbol{S}}$.

The second but last line of the entropy production equation (26) shows that

$$
\frac{\partial \Psi}{\partial \nabla T}=f_{4}(\mathscr{Z}) \nabla \dot{T}
$$

but the free energy density does not depend on $\nabla \dot{T}$, and therefore 


$$
\frac{\partial \Psi}{\partial \nabla T}=0
$$

The free energy density does not depend on temperature gradient.

\subsection{Summary}

The second part of the exploitation of the second law of thermodynamics, namely the conclusions drawn from the entropy production, have considerably restricted the constitutive functions. With the chosen set of variables, Eq. (14) in the domain of the constitutive functions, and including the power of the hyperstress $\underset{\underline{S}}{\boldsymbol{S}}$ in the balance of energy, we found from the exploitation of the second law of thermodynamics that the extra entropy flux $\underline{k}$ and the free energy density $\Psi$ depend solely on the following sets of variables:

$$
\begin{aligned}
& \underline{k}(T, \dot{\boldsymbol{\varepsilon}}), \\
& \Psi(T, \boldsymbol{\varepsilon}, \nabla \boldsymbol{\varepsilon}) .
\end{aligned}
$$

The internal variable or derivatives of it appear neither in the free energy density, nor in the extra entropy flux. In this sense, the inclusion of the internal variable in the beginning does not lead to any modification of the classical theory.

If there is no material anisotropy present (in addition to the possible relevance of tensorial internal variables), there exists no vector function $\underline{k}$ depending on a scalar (the temperature) and a symmetric second-order tensor $(\dot{\varepsilon})$ solely [44]. We conclude from Eq. (39) that the extra entropy flux vanishes, $\underline{k}=0$, i.e., we have the classical relation between entropy flux and heat flux:

$$
\underline{\Phi}=\frac{q}{T} .
$$

The only cause for the entropy flux is the heat flux. There is no contribution from the internal variable, because the internal variable satisfies a pure relaxation equation without a flux.

Our result does not contradict the result of eq. (3.9) in [10], but according to us the constitutive functions are further restricted to the case $\frac{\partial \Psi}{\partial \nabla \alpha}=0$, i.e.,
$B=0$ in the notation of [10], and therefore

$$
\underline{k}=0 .
$$




\subsection{Special case of vanishing hyperstress}

If we had set the hyperstress $\underset{\tilde{S}}{\boldsymbol{S}}$ equal to zero, we could proceed with the evaluation of the fourth line of the entropy production in Eq. (26). This would lead to the conclusion

$$
\begin{aligned}
& \frac{\partial \underline{k}}{\partial \dot{\boldsymbol{\varepsilon}}}=0, \\
& \frac{\varrho}{T} \frac{\partial \Psi}{\partial \nabla_{\boldsymbol{\varepsilon}}}=0 .
\end{aligned}
$$

In this case, the extra entropy flux does not depend on $\dot{\xi}$, but can only depend on temperature, i.e., on a scalar variable. Again, the representation theorem shows that the extra entropy flux vanishes. In addition, the dependence of the free energy is restricted in this case to the equilibrium variables $\Psi(T, \varepsilon)$.

A dependence of the free energy density on the strain gradient is therefore only possible if the power of internal forces is enriched by the introduction of the hyperstress tensor $\boldsymbol{S}$ as suggested in [25]. In the same spirit, more general dependencies of the free energy densities become possible by further extending the power of external forces and introducing additional generalized stress tensors. This is the objective of the next section.

\section{Higher grade theory with enriched power of internal forces}

\subsection{Second gradient and additional degree of freedom theory with state space $\mathscr{Z}_{0}$}

An alternative formulation is presented here for a material theory including the first and second gradients of the displacement field $\underline{u}$, an additional independent variable $\alpha$, and its first gradient $\nabla \alpha$. In the thermomechanical theory, the variables $T$ and $\nabla T$ must also be introduced a priori. The state space is therefore defined as

$$
\mathscr{Z}_{0}=\{T, \boldsymbol{\varepsilon}, \alpha, \nabla T, \nabla \boldsymbol{\varepsilon}, \nabla \alpha\}
$$

It is a subspace of the already introduced state space $\mathscr{Z}$ in Eq. (14). For the sake of conciseness, the Coleman-Noll exploitation procedure of the second law is applied in this section [42]. The Liu approach can be shown to lead to the same conclusions in the present context provided that the Lagrange multiplier $\Lambda^{e}$ is identified with the inverse of $T$. 
3.1.1. Balance equations Following the method of virtual power formulated and applied to first- and second-grade continua in $[26,45,18]$, the overall powers of internal, external, and contact forces in a virtual motion $\vartheta^{\star} \in \mathscr{Z}_{0}$ for a domain $\mathscr{D}$ of the body, are assumed to admit power densities:

$$
\begin{aligned}
& \mathscr{P}^{(i)}\left(\vartheta^{\star} \in \mathscr{Z}_{0}\right)=\int_{\mathscr{D}} p^{(i)}\left(\vartheta^{\star}\right) d V, \quad \mathscr{P}^{(e)}\left(\vartheta^{\star} \in \mathscr{Z}_{0}\right)=\int_{\mathscr{D}} p^{(e)}\left(\vartheta^{\star}\right) d V, \\
& \mathscr{P}^{(c)}\left(\vartheta^{\star} \in \mathscr{Z}_{0}\right)=\int_{\partial \mathscr{D}} p^{(c)}\left(\vartheta^{\star}\right) d S,
\end{aligned}
$$

where $\partial \mathscr{D}$ is the boundary of a subdomain $\mathscr{D}$ of the body.

The power density of internal forces is then taken as a general linear form on the rates of the state variables:

$$
p^{(i)}\left(\vartheta^{\star}\right)=\boldsymbol{\sigma}: \dot{\boldsymbol{\varepsilon}}^{\star}+\underset{\underline{S}}{\vdots}: \nabla \dot{\boldsymbol{\varepsilon}}^{\star}+a \dot{\alpha}^{\star}+\underline{\boldsymbol{b}} \cdot \nabla \dot{\alpha}^{\star}+A \dot{T}^{\star}+\underline{\boldsymbol{B}} \cdot \boldsymbol{\nabla} \dot{T}^{\star} .
$$

Generalized stresses $\boldsymbol{\sigma}, \underline{\boldsymbol{S}}, a, \underline{\boldsymbol{b}}, A, \underline{\boldsymbol{B}}$ are introduced. Objectivity arguments in the absence of rotational degrees of freedom and couple stresses have been used to write the previous form. In particular, it is sufficient to consider a symmetric stress tensor $\boldsymbol{\sigma}$. The introduction of additional contributions to the classical power of internal forces, associated with additional degrees of freedom, represents a systematic approach described in [45] and the references quoted therein. In these references, different examples are provided dealing for instance with the coupling between thermomechanics and electromagnetism. Note that in the present formulation, the temperature itself is raised to the status of an additional degree of freedom following the same scheme. The contributions of $\dot{\alpha}$ and $\nabla \dot{\alpha}$ were added to the classical expression of $p^{(i)}$ in [29] in the case of a damage variable. In the same spirit, the contributions of $\dot{T}, \nabla \dot{T}$ were added in [31] to construct the second-grade thermoelasticity theory of solids. Similarly, the virtual powers of internal and contact forces read:

$$
\begin{aligned}
p^{(e)}\left(\vartheta^{\star}\right)= & \underline{\boldsymbol{f}} \cdot \dot{\boldsymbol{u}}^{\star}+\underset{\boldsymbol{F}}{\boldsymbol{F}}: \dot{\boldsymbol{\varepsilon}}^{\star}+\underset{\underline{\boldsymbol{F}}}{\vdots}: \nabla \dot{\boldsymbol{\varepsilon}}^{\star}+a^{(e)} \dot{\alpha}^{\star} \\
& +\underline{\boldsymbol{b}}^{(e)} \cdot \nabla \dot{\alpha}^{\star}+A^{(e)} \dot{\boldsymbol{T}}^{\star}+\underline{\boldsymbol{B}}^{(e)} \cdot \nabla \dot{T}^{\star}, \\
p^{(c)}\left(\vartheta^{\star}\right)= & \underline{\boldsymbol{t}} \underline{\underline{\boldsymbol{u}}}^{\star}+\underline{\boldsymbol{F}}^{(c)}:\left(D_{n} \dot{\dot{\boldsymbol{u}}}\right)+a^{(c)} \dot{\alpha}^{\star}+A^{(c)} \dot{T}^{\star} .
\end{aligned}
$$

The normal gradient of the velocity field at a surface is called $D_{n} \underline{\dot{\boldsymbol{u}}}$ (see [26] for its definition). Volume forces $\boldsymbol{f}$, volume double and triple forces $\underset{\sim}{\boldsymbol{F}}, \underset{\underline{\boldsymbol{F}}}{\boldsymbol{F}}$, and generalized volume forces $a^{(e)}, \underline{\boldsymbol{b}}^{(e)}, A^{(e)}, \underline{\boldsymbol{B}}^{(e)}$ are introduced for the sake of 
generality ${ }^{1}$. Only the contributions in the virtual power of contact forces that will finally appear in the balance equations are written following arguments put forward in $[26,45]$.

The principle of virtual power then states that the virtual power of externally acting forces is equal to the power of internally acting forces plus the power of acceleration forces:

$$
\mathscr{P}^{(e)}+\mathscr{P}^{(c)}=\mathscr{P}^{(i)}+\int_{\mathscr{D}} \varrho \underline{\ddot{u}} \cdot \underline{\dot{u}}^{\star} d V,
$$

for all virtual fields $\vartheta^{\star}$ with sufficient regularity, and for any subdomain $\mathscr{D}$ of the body. Balance equations and boundary conditions are derived from this principle. The detailed derivation is not given here since it strictly follows the arguments detailed in $[26,45]$. The found balance equations read:

$$
\begin{aligned}
& \boldsymbol{\nabla} \cdot \underline{\boldsymbol{\tau}}+\underline{\boldsymbol{f}}-\varrho \underline{\ddot{u}}=0, \quad \text { with } \underline{\boldsymbol{\tau}}=\boldsymbol{\sigma}-\underset{\boldsymbol{F}}{\boldsymbol{F}}-\boldsymbol{\nabla} \cdot \underline{\boldsymbol{S}}-\boldsymbol{\nabla} \cdot \underline{\boldsymbol{F}}, \\
& \boldsymbol{\nabla} \cdot\left(\underline{\boldsymbol{b}}-\underline{\boldsymbol{b}}^{(e)}\right)-\left(a-a^{(e)}\right)=0, \\
& \boldsymbol{\nabla} \cdot\left(\underline{\boldsymbol{B}}-\underline{\boldsymbol{B}}^{(e)}\right)-\left(A-A^{(e)}\right)=0 .
\end{aligned}
$$

The boundary conditions valid on $\partial \mathscr{D}$ with normal $\underline{\boldsymbol{n}}$ read:

$$
\begin{aligned}
& \underline{\boldsymbol{t}}=\underline{\boldsymbol{\tau}} \cdot \underline{\boldsymbol{n}}+2 R \underline{\boldsymbol{S}}:(\underline{\boldsymbol{n}} \otimes \underline{\boldsymbol{n}})-D_{t}(\underline{\boldsymbol{S}} \cdot \underline{\boldsymbol{n}}), \\
& \underline{\boldsymbol{F}}^{(c)}=\underline{\boldsymbol{S}}:(\underline{\boldsymbol{n}} \otimes \underline{\boldsymbol{n}}), \\
& a^{(c)}=\left(\underline{\boldsymbol{b}}-\underline{\boldsymbol{b}}^{(e)}\right) \cdot \underline{\boldsymbol{n}}, \\
& A^{(c)}=\left(\underline{\boldsymbol{B}}-\underline{\boldsymbol{B}}^{(e)}\right) \cdot \underline{\boldsymbol{n}} .
\end{aligned}
$$

In the classical theory, the vectors $\underline{\boldsymbol{b}}$ and $\underline{\boldsymbol{B}}$ do not exist. As a result of the previous balance equations, the stresses $a$ and $A$ must then vanish also. The classical balance of momentum equation is then retrieved.

The balance of internal energy introduces the density of internal energy $e\left(\mathscr{Z}_{0}\right)$. It reads

$$
\varrho \dot{e}=p^{(i)}-\nabla \cdot \underline{\boldsymbol{q}},
$$

where the power of internal forces is defined by (48).

\footnotetext{
${ }^{1}$ Volume couples could also have been introduced but, for that purpose, the skew-symmetric part of the velocity gradient should have been introduced in the state space $\mathscr{Z}_{0}$. As a result, the volume double forces are represented here only by a symmetric second-order tensor $\boldsymbol{F}$. 
The entropy inequality still reads

$$
\varrho \dot{\eta}+\nabla \cdot \underline{\boldsymbol{\Phi}} \geq 0, \quad \text { with } \underline{\boldsymbol{\Phi}}=\frac{\underline{\boldsymbol{q}}}{T}+\underline{\boldsymbol{k}} .
$$

The extra entropy flux $\underline{\boldsymbol{k}}$ also operates on the state space $\mathscr{Z}_{0}$.

3.1.2. Exploitation of the second law according to Coleman-Noll In the thermomechanics of solids, the Helmholtz free energy density

$$
\Psi\left(\mathscr{Z}_{0}\right) \equiv \Psi(T, \boldsymbol{\varepsilon}, \alpha, \nabla T, \nabla \boldsymbol{\varepsilon}, \nabla \alpha)=\varrho e-\varrho T \eta
$$

is introduced with the indicated dependence. The entropy production $\sigma$ takes then the following form:

$$
\sigma T \equiv p^{(i)}-\varrho \dot{\Psi}-\varrho \eta \dot{T}-\frac{1}{T} \underline{\boldsymbol{q}} \cdot \boldsymbol{\nabla} T+T \nabla \cdot \underline{\boldsymbol{k}} \geq 0 .
$$

When the explicit dependence of the constitutive functions on the state variables $\mathscr{Z}_{0}$ and the expression (48) of the enriched power of internal forces are taken into account, the previous inequality results in a generalized ClausiusDuhem inequality:

$$
\begin{aligned}
(\boldsymbol{\sigma} & \left.-\varrho \frac{\partial \Psi}{\partial \underline{\boldsymbol{\varepsilon}}}\right): \dot{\boldsymbol{\varepsilon}}+\left(\underset{\underline{S}}{\boldsymbol{S}}-\varrho \frac{\partial \Psi}{\partial \nabla \boldsymbol{\varepsilon}}\right) \vdots \nabla \dot{\boldsymbol{\varepsilon}} \\
& +\left(A-\varrho \frac{\partial \Psi}{\partial T}-\varrho \eta\right) \dot{T}+\left(\underline{\boldsymbol{B}}-\varrho \frac{\partial \Psi}{\partial \boldsymbol{\nabla} T}\right) \cdot \boldsymbol{\nabla} \dot{T} \\
& +\left(a-\varrho \frac{\partial \Psi}{\partial \alpha}\right) \dot{\alpha}+\left(\underline{\boldsymbol{b}}-\varrho \frac{\partial \Psi}{\partial \boldsymbol{\nabla} \alpha}\right) \cdot \nabla \dot{\alpha}+T \nabla \cdot \underline{\boldsymbol{k}}-\frac{1}{T} \underline{\boldsymbol{q}} \cdot \boldsymbol{\nabla} T \geq 0
\end{aligned}
$$

Since $\dot{T}, \dot{\boldsymbol{\xi}}, \dot{\alpha}, \nabla \dot{T}, \nabla \dot{\boldsymbol{\xi}}, \nabla \dot{\alpha}$ do not belong to the state space $\mathscr{Z}_{0}$, the following state laws can be derived:

$$
\begin{aligned}
& \boldsymbol{\sigma}=\varrho \frac{\partial \Psi}{\partial \underline{\varepsilon}}, \quad \boldsymbol{S}=\varrho \frac{\partial \Psi}{\partial \boldsymbol{\nabla} \boldsymbol{\varepsilon}}, \\
& \varrho \eta=-\varrho \frac{\partial \Psi}{\partial T}+A, \quad a=\varrho \frac{\partial \Psi}{\partial \alpha}, \\
& \underline{\boldsymbol{B}}=\varrho \frac{\partial \Psi}{\partial \boldsymbol{\nabla} T}, \quad \underline{\boldsymbol{b}}=\varrho \frac{\partial \Psi}{\partial \boldsymbol{\nabla} \alpha}
\end{aligned}
$$


As a result, the second law does not restrict the number of state variables on which the free energy density can depend. In particular, it can be an explicit function of the strain gradient $\nabla \boldsymbol{\varepsilon}$, of the gradient of the additional variable $\nabla \alpha$, and of the temperature gradient $\nabla T$. The former possibility was exploited in [29] to construct a gradient of damage theory. The latter possibility was exploited in [31] to formulate linear thermoelasticity constitutive equations for second-grade media.

3.1.3. Exploitation of the residual inequality After taking the state laws into account, the residual entropy production inequality is

$$
\sigma=\nabla \cdot \underline{k}-\frac{1}{T^{2}} \underline{\boldsymbol{q}} \cdot \nabla T \geq 0
$$

Since $\underline{\boldsymbol{k}}$ a priori is a constitutive function of all state variables $\mathscr{Z}_{0}$, the previous inequality becomes

$$
\begin{aligned}
\frac{\partial \underline{\boldsymbol{k}}}{\partial T} . \boldsymbol{\nabla} T+\frac{\partial \underline{\boldsymbol{k}}}{\partial \underline{\boldsymbol{\varepsilon}}}: \nabla \boldsymbol{\varepsilon}+\frac{\partial \underline{\boldsymbol{k}}}{\partial \alpha} \cdot \nabla \alpha+\frac{\partial \underline{\boldsymbol{k}}}{\partial \boldsymbol{\nabla} T}: \nabla \nabla T \\
+\frac{\partial \underline{\boldsymbol{k}}}{\partial \boldsymbol{\nabla} \boldsymbol{\varepsilon}}:: \nabla \nabla \underline{\boldsymbol{\varepsilon}}+\frac{\partial \underline{\boldsymbol{k}}}{\partial \boldsymbol{\nabla} \alpha}: \nabla \nabla \alpha-\frac{1}{T^{2}} \underline{\boldsymbol{q}} . \nabla T \geq 0 .
\end{aligned}
$$

The following relations can then be deduced from the fact that $\nabla \nabla T, \nabla \nabla \boldsymbol{\varepsilon}$, $\nabla \nabla \alpha$ do not belong to $\mathscr{Z}_{0}$ :

$$
\frac{\partial \underline{\boldsymbol{k}}}{\partial \boldsymbol{\nabla} T}=\frac{\partial \underline{\boldsymbol{k}}}{\partial \boldsymbol{\nabla} \alpha}=0, \quad \frac{\partial \underline{\boldsymbol{k}}}{\partial \boldsymbol{\nabla} \boldsymbol{\varepsilon}}=0
$$

The inequality is now exploited further making use of Eq. (7). The partial derivatives of $\boldsymbol{k}$ with respect to $T, \varepsilon, \alpha$ are then homogeneous functions in $\nabla T$, $\nabla \varepsilon, \nabla \alpha$, respectively:

$$
\begin{aligned}
& \frac{\partial \underline{\boldsymbol{k}}}{\partial T}-\frac{1}{T^{2}} \underline{\boldsymbol{q}}=f_{q}\left(\mathscr{Z}_{0}\right) \cdot \nabla T \\
& \frac{\partial \underline{\boldsymbol{k}}}{\partial \boldsymbol{\varepsilon}}=f_{\varepsilon}\left(\mathscr{Z}_{0}\right) \vdots \nabla \boldsymbol{\varepsilon} \\
& \frac{\partial \underline{\boldsymbol{k}}}{\partial \alpha}=f_{\alpha}\left(\mathscr{Z}_{0}\right) \cdot \nabla \alpha
\end{aligned}
$$


where the constitutive tensor functions $f_{q}, f_{\varepsilon}, f_{\alpha}$ of the state variables are formally introduced. However, the left-hand side of Eq. (71) does not depend on $\nabla \varepsilon$ according to Eq. (69). The only possibility is therefore $f_{\varepsilon} \equiv 0$. Similar arguments hold for the dependence on $\nabla \alpha$, so that $f_{\alpha}$ must vanish. As a result, the extra-entropy flux can only be a function $\underline{\boldsymbol{k}}(T)$. In the isotropic case, $\underline{\boldsymbol{k}}$ must therefore vanish.

\subsection{Second gradient and additional degree of freedom theory with variables $\mathscr{Z}$}

If the previous state space is extended to include the rates of the fundamental variables $(T, \varepsilon, \alpha)$ and of their gradients, and so to coincide with state space $\mathscr{Z}$ of Eq. (14), modifications must be made to the previous theory of gradientdependent thermomechanical theory.

The expressions of the enriched powers of internal, external, and contact forces (48) to (50) are assumed to remain unchanged because second-time derivatives of the fundamental variables are a priori excluded of these power densities. As a result, the balance equations and boundary conditions (52) to (58) still hold. The differences will arise in the functional dependence of the constitutive functions $\Psi$ and $\underline{\boldsymbol{k}}$ on the extended state space.

3.2.1. Exploitation of the second law according to Coleman-Noll The constitutive functions

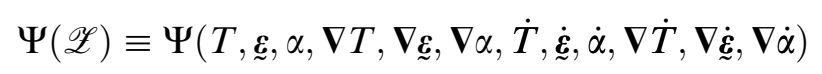

and $\underline{\boldsymbol{k}}(\mathscr{Z})$ are introduced in the entropy inequality (60). This gives

$$
\begin{aligned}
& \left(\boldsymbol{\sigma}-\varrho \frac{\partial \Psi}{\partial \boldsymbol{\varepsilon}}\right): \dot{\boldsymbol{\varepsilon}}+\left(\underline{\underline{\boldsymbol{S}}}-\varrho \frac{\partial \Psi}{\partial \nabla \underline{\boldsymbol{\varepsilon}}}+T \frac{\partial \underline{\boldsymbol{k}}}{\partial \dot{\boldsymbol{\varepsilon}}}\right) \vdots \nabla \dot{\boldsymbol{\varepsilon}} \\
& +\left(A-\varrho \frac{\partial \Psi}{\partial T}-\varrho \eta\right) \dot{T}+\left(\underline{\boldsymbol{B}}-\varrho \frac{\partial \Psi}{\partial \boldsymbol{\nabla} T}+T \frac{\partial \underline{\boldsymbol{k}}}{\partial \dot{T}}\right) \cdot \nabla \dot{T} \\
& +\left(a-\varrho \frac{\partial \Psi}{\partial \alpha}\right) \dot{\alpha}+\left(\underline{b}-\varrho \frac{\partial \Psi}{\partial \nabla \alpha}+T \frac{\partial \underline{\boldsymbol{k}}}{\partial \dot{\alpha}}\right) \cdot \nabla \dot{\alpha} \\
& +\varrho \frac{\partial \Psi}{\partial \dot{T}} \ddot{T}+\varrho \frac{\partial \Psi}{\partial \dot{\boldsymbol{\varepsilon}}}: \ddot{\boldsymbol{\varepsilon}}+\varrho \frac{\partial \Psi}{\partial \dot{\alpha}} \ddot{\alpha} \\
& +\varrho \frac{\partial \Psi}{\partial \nabla \dot{T}} \cdot \nabla \ddot{T}+\varrho \frac{\partial \Psi}{\partial \nabla \dot{\varepsilon}} \vdots \nabla \ddot{\varepsilon}+\varrho \frac{\partial \Psi}{\partial \nabla \dot{\alpha}} \cdot \nabla \ddot{\alpha}+T \sigma-\frac{1}{T} \underline{\boldsymbol{q}} \cdot \nabla T \geq 0 .
\end{aligned}
$$


The entropy production $\sigma$ will be given explicitly in Section 3.2.2. The variables $\ddot{T}, \ddot{\boldsymbol{\varepsilon}}, \ddot{\alpha}, \nabla \ddot{T}, \nabla \ddot{\boldsymbol{\varepsilon}}, \nabla \ddot{\alpha}$ do not belong to the state space $\mathscr{Z}$, so that the following conditions are necessary to fulfill the previous inequality:

$$
\begin{aligned}
& \frac{\partial \Psi}{\partial \dot{T}}=\frac{\partial \Psi}{\partial \dot{\alpha}}=0, \quad \frac{\partial \Psi}{\partial \dot{\varepsilon}}=0, \\
& \frac{\partial \Psi}{\partial \nabla \dot{T}}=\frac{\partial \Psi}{\partial \nabla \dot{\alpha}}=0, \quad \frac{\partial \Psi}{\partial \nabla \dot{\varepsilon}}=0 .
\end{aligned}
$$

Accordingly, the constitutive dependence of the free energy on the state variable is reduced to

$$
\Psi(T, \underline{\varepsilon}, \alpha, \nabla T, \nabla \boldsymbol{\varepsilon}, \nabla \alpha)=\Psi\left(\mathscr{Z}_{0}\right)
$$

The entropy inequality can be exploited further making use of the condition (7). There must exist constitutive functions $f_{\sigma}, f_{S}, f_{\eta}, f_{b}, f_{a}, f_{B}$ of the state variables $\mathscr{Z}$ such that

$$
\begin{aligned}
& \boldsymbol{\sigma}-\varrho \frac{\partial \Psi}{\partial \boldsymbol{\Psi}}=f_{\sigma}(\mathscr{Z}): \dot{\boldsymbol{\varepsilon}}, \\
& \underset{\boldsymbol{S}}{\underline{\boldsymbol{s}}}-\varrho \frac{\partial \Psi}{\partial \boldsymbol{\nabla} \boldsymbol{\varepsilon}}+T \frac{\partial \underline{\boldsymbol{k}}}{\partial \dot{\boldsymbol{\varepsilon}}}=f_{S}(\mathscr{Z}) \vdots \nabla \dot{\boldsymbol{\varepsilon}} \\
& A-\varrho \frac{\partial \Psi}{\partial T}-\varrho \eta=f_{\eta}(\mathscr{Z}) \dot{T} \\
& a-\varrho \frac{\partial \Psi}{\partial \alpha}=f_{a}(\mathscr{Z}) \dot{\alpha} \\
& \underline{\boldsymbol{B}}-\varrho \frac{\partial \Psi}{\partial \boldsymbol{\nabla} T}+T \frac{\partial \underline{\boldsymbol{k}}}{\partial \dot{T}}=f_{B}(\mathscr{Z}) . \nabla \dot{T}, \\
& \underline{\boldsymbol{b}}-\varrho \frac{\partial \Psi}{\partial \boldsymbol{\nabla} \alpha}+T \frac{\partial \underline{\boldsymbol{k}}}{\partial \dot{\alpha}}=f_{b}(\mathscr{Z}) . \nabla \dot{\alpha} .
\end{aligned}
$$

In the special case for which the stress functions $\boldsymbol{\sigma}, \underset{\tilde{S}}{\boldsymbol{S}}, \boldsymbol{a}, \underline{\boldsymbol{b}}, A, \underline{\boldsymbol{B}}$ do not depend on the rates $\dot{T}, \dot{\xi}, \dot{\alpha}, \nabla \dot{T}, \nabla \dot{\boldsymbol{\varepsilon}}, \nabla \dot{\alpha}$, the state laws (64) to (66) are retrieved. But the previous forms (78) to (83) remain the most general admissible ones for the state space $\mathscr{Z}$. 
3.2.2. Exploitation of the residual inequality The residual entropy production contains the following contributions of the extra-entropy flux:

$$
\begin{aligned}
\sigma= & \frac{\partial \underline{\boldsymbol{k}}}{\partial T} \cdot \boldsymbol{\nabla} T+\frac{\partial \underline{\boldsymbol{k}}}{\partial \boldsymbol{\varepsilon}}: \nabla \underline{\boldsymbol{\varepsilon}}+\frac{\partial \underline{\boldsymbol{k}}}{\partial \alpha} \cdot \nabla \alpha \\
& +\frac{\partial \underline{\boldsymbol{k}}}{\partial \boldsymbol{\nabla} T}: \nabla \boldsymbol{\nabla} T+\frac{\partial \underline{\boldsymbol{k}}}{\partial \boldsymbol{\nabla} \boldsymbol{\varepsilon}}:: \nabla \nabla \boldsymbol{\varepsilon}+\frac{\partial \underline{\boldsymbol{k}}}{\partial \boldsymbol{\nabla} \alpha}: \nabla \nabla \alpha \\
& +\frac{\partial \underline{\boldsymbol{k}}}{\partial \boldsymbol{\nabla} \dot{T}}: \nabla \nabla \dot{T}+\frac{\partial \underline{\boldsymbol{k}}}{\partial \boldsymbol{\nabla} \dot{\boldsymbol{\varepsilon}}}:: \nabla \nabla \dot{\boldsymbol{\varepsilon}}+\frac{\partial \underline{\boldsymbol{k}}}{\partial \boldsymbol{\nabla} \dot{\alpha}}: \nabla \nabla \dot{\alpha} .
\end{aligned}
$$

Since neither the variables $\nabla \nabla T, \nabla \nabla \boldsymbol{\varepsilon}, \nabla \nabla \alpha$, nor $\nabla \nabla \dot{T}, \nabla \nabla \dot{\varepsilon}, \nabla \nabla \dot{\alpha}$ belong to $\mathscr{Z}$, the following partial derivatives must vanish:

$$
\begin{gathered}
\frac{\partial \underline{\boldsymbol{k}}}{\partial \boldsymbol{\nabla} T}=\frac{\partial \underline{\boldsymbol{k}}}{\partial \boldsymbol{\nabla} \alpha}=0, \quad \frac{\partial \underline{\boldsymbol{k}}}{\partial \boldsymbol{\nabla} \boldsymbol{\varepsilon}}=0, \\
\frac{\partial \underline{\boldsymbol{k}}}{\partial \boldsymbol{\nabla} \dot{T}}=\frac{\partial \underline{\boldsymbol{k}}}{\partial \nabla \dot{\alpha}}=0, \quad \frac{\partial \underline{\boldsymbol{k}}}{\partial \boldsymbol{\nabla} \dot{\boldsymbol{\varepsilon}}}=0 .
\end{gathered}
$$

Constitutive functions $f_{q}, f_{\varepsilon}, f_{\alpha}$ of the state variables may then exist such that the Eqs. (70) to (72) still hold. The same reasoning as in Section 3.1.3 leads to the conclusion that

$$
\frac{\partial \underline{\boldsymbol{k}}}{\partial \underline{\boldsymbol{\varepsilon}}}=0, \quad \frac{\partial \underline{\boldsymbol{k}}}{\partial \alpha}=0
$$

Accordingly, the extra-entropy flux is reduced to a function

$$
\underline{\boldsymbol{k}}(T, \dot{T}, \dot{\boldsymbol{\varepsilon}}, \dot{\alpha}) \text {. }
$$

3.2.3. Summary The introduction of generalized stresses associated to the strain gradient, gradient of an additional degree of freedom, and temperature gradient into an enriched power of internal forces makes it possible to envisage general constitutive functions of the form

$$
\Psi(T, \boldsymbol{\varepsilon}, \alpha, \nabla T, \nabla \boldsymbol{\varepsilon}, \nabla \alpha), \quad \underline{\boldsymbol{k}}(T, \dot{T}, \dot{\boldsymbol{\varepsilon}}, \dot{\alpha}) .
$$

In contrast to the more classical case handled in Section 2, an explicit dependence of the free energy density on the gradient of an additional variable $\alpha$ 
and on the temperature gradient is possible. This has proved to be necessary in a damage gradient theory of materials [29] and in the thermoelasticity of second-grade media [31].

The next section gives an alternative way of allowing dependences of the form (89) without enriching explicitly the power of internal forces.

\section{Nonclassical theory with an additional variable satisfying a balance equation}

We will consider again the case of a material where an additional variable $\alpha$ is relevant for the description of complex material behavior. In our notation, this is a scalar variable, but the generalization to a tensorial variable or to a set of several variables is straightforward. Now we introduce a flux of the additional variable, i.e., we assume a transport of the variable and its differential equation is an evolution-diffusion equation and not a pure relaxation equation.

\subsection{Results from the exploitation of the dissipation inequality according to Liu}

The balance equations of mass, momentum, and energy remain unchanged:

Balance of mass:

$$
\grave{\varrho}+\varrho \nabla \cdot \underline{v}=0
$$

\section{Balance of momentum:}

$$
\varrho \underline{\dot{v}}-\nabla \cdot(\boldsymbol{\sigma}-\nabla \cdot \underline{\boldsymbol{S}})-\varrho \underline{f}=0 .
$$

\section{Balance of internal energy:}

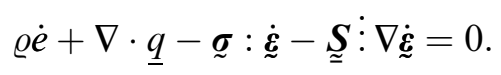

For the additional variable, we have now an evolution-diffusion equation

$$
\frac{d \alpha}{d t}+\nabla \cdot \underline{J}_{\alpha}=\pi_{\alpha}
$$

with a flux term $\underline{J}_{\alpha}$. 
Consequently, the following inequality has to be exploited:

$$
\begin{aligned}
\varrho \dot{\eta}+ & \nabla \cdot \underline{\Phi}+\Lambda^{\varrho}(\varrho \dot{\varrho}+\varrho \nabla \cdot \underline{v})+\underline{\Lambda}^{v}(\varrho \underline{\dot{v}}-\nabla \cdot(\boldsymbol{\sigma}+\nabla \cdot \underline{\underline{S}})-\varrho \underline{f}) \\
& +\Lambda^{e}(\varrho \dot{\boldsymbol{e}}+\nabla \cdot \underline{q}-\underline{\tau}: \dot{\boldsymbol{\varepsilon}}-\underline{\underline{S}} \vdots \nabla \underline{\dot{\boldsymbol{\varepsilon}}})+\Lambda^{\alpha}\left(\dot{\alpha}+\nabla \cdot \underline{J}_{\alpha}-\pi_{\alpha}\right) \geq 0 .
\end{aligned}
$$

The set of relevant variables for the constitutive functions is supposed to be the same as in Section 2:

$$
\mathscr{Z}=\{T, \boldsymbol{\xi}, \alpha, \nabla T, \nabla \boldsymbol{\varepsilon}, \nabla \alpha, \dot{T}, \nabla \dot{T}, \dot{\boldsymbol{\varepsilon}}, \nabla \dot{\boldsymbol{\varepsilon}}, \dot{\alpha}, \nabla \dot{\alpha}\}
$$

The multiplier $\Lambda^{e}$ is identified as $-1 / T$, and $\underline{\Lambda}^{v}$ is calculated as $\underline{\Lambda}^{v}=0$. The remaining inequality is linear in the higher derivatives

$$
\ddot{T}, \nabla \ddot{T}, \ddot{\boldsymbol{\varepsilon}}, \nabla \ddot{\boldsymbol{\varepsilon}}, \ddot{\alpha}, \nabla \ddot{\alpha}, \nabla \nabla T, \nabla \nabla \boldsymbol{\varepsilon}, \nabla \nabla \alpha, \nabla \nabla \dot{T}, \nabla \nabla \dot{\boldsymbol{\varepsilon}}, \nabla \nabla \dot{\alpha},
$$

and each of them leads to a restriction on constitutive functions. The first set of equations, corresponding to the higher derivatives $\ddot{T}, \nabla \ddot{T}, \ddot{\boldsymbol{\varepsilon}}, \nabla \ddot{\boldsymbol{\varepsilon}}, \ddot{\alpha}, \nabla \ddot{\alpha}$, leads to restrictions for the free energy density $\Psi=e-T \eta$ :

$$
\frac{\partial \Psi}{\partial v_{i}}=0 \quad \text { for } v_{i} \in(\dot{T}, \nabla \dot{T}, \dot{\dot{\varepsilon}}, \nabla \dot{\boldsymbol{\varepsilon}}, \dot{\alpha}, \nabla \dot{\alpha})
$$

i.e., the free energy density is a function of the variables:

$$
\Psi(T, \boldsymbol{\varepsilon}, \alpha, \nabla T, \nabla \boldsymbol{\varepsilon}, \nabla \alpha)
$$

The next set of equations, corresponding to the higher derivatives $\nabla \nabla T, \nabla \nabla \varepsilon$, $\nabla \nabla \alpha, \nabla \nabla \dot{T}, \nabla \nabla \dot{\varepsilon}, \nabla \nabla \dot{\alpha}$, are relations between the extra entropy flux $\underline{k}$ and the flux of the additional variable $\underline{J}_{\alpha}$ :

$$
\frac{\partial \underline{k}}{\partial w_{i}}=-\Lambda^{\alpha} \frac{\partial \underline{J}_{\alpha}}{\partial w_{i}} \quad \text { for } w_{i} \in(\nabla T, \nabla \boldsymbol{\varepsilon}, \nabla \alpha, \nabla \dot{T}, \nabla \dot{\boldsymbol{\varepsilon}}, \nabla \dot{\alpha})
$$

One of these relations may be used to calculate $\Lambda^{\alpha}$, but they do not allow any further conclusions concerning the extra entropy flux.

\subsection{Exploitation of the residual inequality}

The resulting expression for the entropy production (which is a state space function, not involving any higher derivatives) is given in the form of a formal scalar product: 


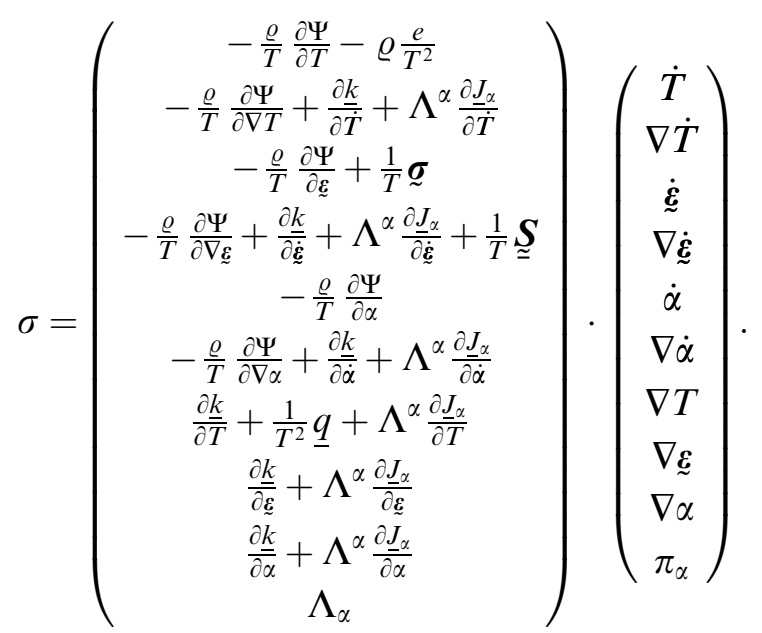

Now the last term in the entropy production (100) contains the production of the additional variable $\pi_{\alpha}$. This is a constitutive quantity, depending in general on all state space variables. Therefore, not all quantities in the right column, Eq. (100), are independent, but $\pi_{\alpha}$ may depend on all other quantities in the column. Therefore, the entropy production cannot be exploited further in this case. We end up with the restrictions derived from the Liu procedure, and the extra entropy flux can be non-zero.

\subsection{Summary}

Introducing an additional variable with an evolution-diffusion equation of motion, the results on constitutive functions from the second law of thermodynamics are much less restrictive than in the case of an internal variable obeying a relaxation equation, especially as we cannot conclude that the extra entropy flux $\underline{k}$ vanishes.

\section{Higher gradient theories}

We treat now the general case of an $n$-gradient theory, i.e., gradients of strain up to order $n$ are included in the domain of constitutive functions $\mathscr{Z}_{1}$. In addition, the possibility of first-order time derivatives of these deformation variables being relevant for material behavior is taken into consideration:

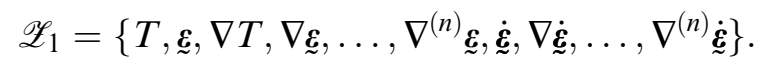

It is assumed that the balance of momentum (9) is unchanged. In particular, no stress tensors of higher order than $\underset{\underline{S}}{\boldsymbol{S}}$ are introduced. Internal variables are not taken into account here for the sake of simplicity. 
The higher derivatives are in this case:

$$
\dot{T}, \nabla \dot{T}, \ddot{\boldsymbol{\varepsilon}}, \nabla \ddot{\boldsymbol{\varepsilon}}, \ldots, \nabla^{(n)} \ddot{\boldsymbol{\xi}}, \nabla \nabla T, \nabla^{(n+1)} \boldsymbol{\varepsilon}, \nabla^{(n+1)} \dot{\boldsymbol{\varepsilon}}, \underline{\underline{v}}
$$

Corresponding to each higher derivative, there is an equation restricting constitutive functions. From this set of equations, the quantities $\Lambda^{v}$ and $\Lambda^{e}$ are eliminated. Again we introduce the abbreviations free energy $\Psi:=e-T \eta$ and the extra entropy flux $\underline{k}=\underline{\Phi}-\frac{q}{T}$.

$\Lambda^{e}$ is identified with the inverse temperature:

$$
\Lambda^{e}=\frac{1}{T}
$$

From the equation corresponding to the higher derivative $\underline{\dot{v}}$, we calculate:

$$
\underline{\dot{v}}: \underline{\Lambda^{v}}=0
$$

$\Lambda^{\varrho}$ cannot be calculated, but is not needed in the following, because neither the restrictions on constitutive functions, nor the entropy production depend on $\Lambda^{\varrho}$.

The remaining restrictions on constitutive functions from the second law are:

$$
\begin{aligned}
& \frac{\partial \Psi}{\partial T}=-\frac{e}{T} \\
& \frac{\partial \Psi}{\partial z_{i}}=0, \quad z_{i} \in\left\{\nabla T, \dot{\boldsymbol{\varepsilon}}, \nabla \dot{\boldsymbol{\varepsilon}}, \ldots, \nabla^{(n)} \dot{\boldsymbol{\varepsilon}}\right\} \\
& \frac{\partial \underline{k}}{\partial u_{i}}=0, \quad u_{i} \in\left\{\nabla T, \nabla^{(n)} \boldsymbol{\varepsilon}, \nabla^{(n)} \dot{\boldsymbol{\varepsilon}}\right\}
\end{aligned}
$$

In summary, the exploitation of the second law with the balance equations as constraints has restricted the dependence of the extra entropy flux and of the free energy density to the following variables:

$$
\begin{aligned}
& \underline{k}\left(T, \boldsymbol{\xi}, \nabla \boldsymbol{\varepsilon}, \ldots, \nabla^{(n-1)} \boldsymbol{\xi}, \dot{\boldsymbol{\varepsilon}}, \nabla \dot{\boldsymbol{\varepsilon}}, \ldots, \nabla^{(n-1)} \dot{\boldsymbol{\varepsilon}}\right) \\
& \Psi\left(T, \boldsymbol{\xi}, \nabla \boldsymbol{\varepsilon}, \ldots, \nabla^{(n)} \boldsymbol{\varepsilon}\right) .
\end{aligned}
$$

The free energy density does not depend on any time derivative. 
The entropy production, which is a function of the state space variables, reads in this example:

$$
\begin{aligned}
& \sigma=\left(-\frac{\varrho}{T} \frac{\partial \Psi}{\partial \boldsymbol{\xi}}+\frac{1}{T} \boldsymbol{\sigma}\right): \dot{\boldsymbol{\varepsilon}}+\left(-\frac{\varrho}{T} \frac{\partial \Psi}{\partial \nabla \underline{\boldsymbol{\varepsilon}}}+\frac{\partial \underline{\underline{\boldsymbol{k}}}}{\partial \dot{\boldsymbol{\varepsilon}}}+\frac{1}{T} \underset{\underline{\boldsymbol{S}}}{\boldsymbol{\boldsymbol { \tau }}}\right) \vdots \nabla \dot{\boldsymbol{\varepsilon}} \\
& +\left(-\frac{\varrho}{T} \frac{\partial \Psi}{\partial \nabla \nabla \boldsymbol{\varepsilon}}+\frac{\partial \underline{\underline{k}}}{\partial \dot{\nabla} \boldsymbol{\xi}}\right):: \nabla \nabla \dot{\boldsymbol{\varepsilon}} \\
& +\cdots+\left(-\frac{\varrho}{T} \frac{\partial \Psi}{\partial \nabla^{(n)} \boldsymbol{\varepsilon}}+\frac{\partial \underline{\boldsymbol{k}}}{\partial \nabla^{(n-1)} \dot{\boldsymbol{\varepsilon}}}\right) \cdot{ }^{(n+2)} \nabla^{(n)} \dot{\boldsymbol{\varepsilon}} \\
& +\left(\frac{\partial \underline{k}}{\partial T}+\frac{1}{T^{2}} \underline{q}\right) \cdot \nabla T+\frac{\partial \underline{k}}{\partial \underline{\boldsymbol{\varepsilon}}}: \nabla \underline{\boldsymbol{\varepsilon}}+\frac{\partial \underline{k}}{\partial \nabla \underline{\boldsymbol{\varepsilon}}}:: \nabla \nabla \underline{\mathcal{E}} \\
& +\cdots+\frac{\partial \underline{\underline{k}}}{\partial \nabla^{(n-1)} \boldsymbol{\varepsilon}} \cdot{ }^{(n+2)} \nabla^{(n)} \boldsymbol{\varepsilon} \geq 0 .
\end{aligned}
$$

This inequality is exploited now further making use of Eq. (7).

\subsection{Exploitation of the residual inequality}

The entropy production is written in the form of a formal scalar product:

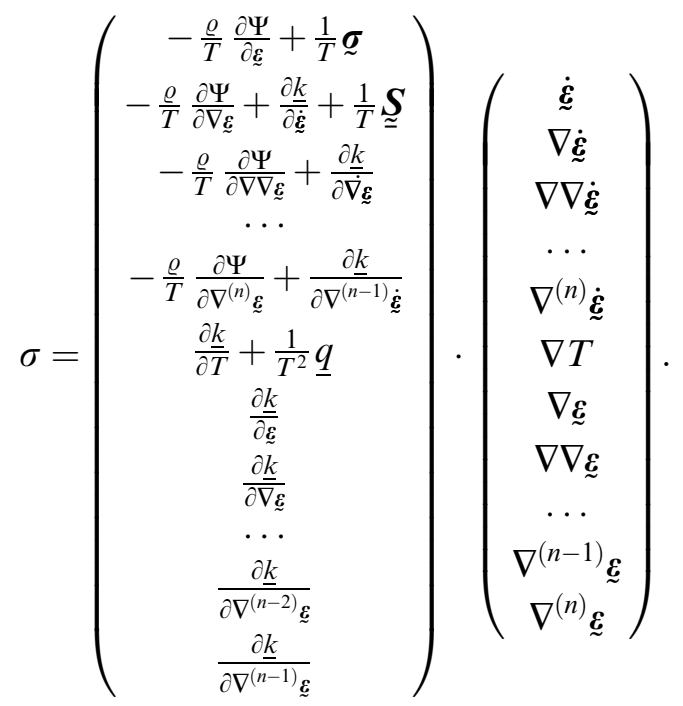

Again we have found the entropy production in the form of a sum of products of state space variables and derivatives of constitutive functions. Under the presupposition that the dependence of the constitutive functions on the 
state space variables is continuous, it follows according to Eq. (7) that each constitutive quantity in the row at the left-hand side is a homogeneous function of the state space variable in the row at the right-hand side in the same line. This fact is exploited now, beginning in the last line of Eq. (111):

$$
\frac{\partial \underline{k}}{\partial \nabla^{(n-1)} \boldsymbol{\varepsilon}}=f_{13}\left(\mathscr{Z}_{1}\right) \nabla^{(n)} \boldsymbol{\varepsilon}
$$

where $f_{13}$ is up to now an arbitrary function of the state space variables. On the other hand, $\underline{k}$ does not depend on $\nabla^{(n)} \boldsymbol{\varepsilon}$ as a consequence of the dissipation inequality (see Eq. [108]) and therefore, the left-hand side of Eq. (112), $\frac{\partial \underline{k}}{\partial \nabla^{(n-1)} \varepsilon}$, is also independent of $\nabla^{(n)} \varepsilon$. Consequently, the only possibility to fulfill Eq. (112) is:

$$
f_{13}=0 \Rightarrow \frac{\partial \underline{\underline{k}}}{\partial \nabla^{(n-1)} \boldsymbol{\varepsilon}}=0
$$

The extra entropy flux does not depend on the $(n-1)$-order gradient of strain. The exploitation of the entropy production proceeds with the line above in Eq. (111). This shows analogously that $\underline{k}$ does not depend on the $(n-2)$ order gradient of strain:

$$
\frac{\partial \underline{k}}{\partial \nabla^{(n-2)} \boldsymbol{\varepsilon}}=f_{23}\left(\mathscr{Z}_{1}\right) \nabla^{(n-1)} \boldsymbol{\varepsilon},
$$

where $f_{23}$ is up to now an arbitrary function of the state space variables. On the other hand, $\underline{k}$ does not depend on $\nabla^{(n-1)} \boldsymbol{\varepsilon}$ according to the previous conclusion [see Eq. (113)] and therefore, the left-hand side of Eq. (114), $\frac{\partial \underline{k}}{\partial \nabla^{(n-2)} \varepsilon}$, is also independent of $\nabla^{(n-1)} \xi$, and the only way to fulfill Eq. (114) is:

$$
f_{23}=0 \Rightarrow \frac{\partial \underline{k}}{\partial \nabla^{(n-2)} \boldsymbol{\varepsilon}}=0 .
$$

The successive exploitation of the lines in the entropy production (111) shows that $\underline{k}$ does not depend on any gradient of strain up to the conclusion

$$
\frac{\partial \underline{k}}{\partial \underline{\varepsilon}}=0
$$

Concerning the dependence of the extra entropy flux $\underline{k}$ on temperature, we cannot draw any conclusion from the entropy production, because the heat 
flux $q$ in the previous line in Eq. (111) is not restricted by the dissipation inequality.

The results on the extra entropy flux and the free energy density derived up to here can be summarized as:

$$
\underline{k}\left(T, \dot{\boldsymbol{\varepsilon}}, \dot{\nabla} \boldsymbol{\varepsilon}, \ldots, \nabla^{(n-1)} \dot{\boldsymbol{\varepsilon}}\right) .
$$

Apart from temperature, the extra entropy flux depends only on time derivatives, whereas

$$
\Psi\left(T, \boldsymbol{\varepsilon}, \nabla \boldsymbol{\varepsilon}, \ldots, \nabla^{(n)} \boldsymbol{\varepsilon}\right)
$$

does not depend on any time derivative.

Further conclusions are drawn from the exploitation of the remaining lines of the entropy production. The next line in Eq. (111) results in

$$
-\frac{\varrho}{T} \frac{\partial \Psi}{\partial \nabla^{(n)} \boldsymbol{\xi}}+\frac{\partial \underline{k}}{\partial \nabla^{(n-1)} \dot{\boldsymbol{\varepsilon}}}=f_{33}\left(\mathscr{Z}_{1}\right) \nabla^{(n)} \dot{\boldsymbol{\varepsilon}}
$$

with an arbitrary state space function $f_{33}$.

$\Psi$ and $\underline{k}$ both do not depend on $\nabla^{(n)} \dot{\boldsymbol{\varepsilon}}$, and therefore the right-hand side of Eq. (119) is independent of $\nabla^{(n)} \dot{\boldsymbol{\varepsilon}}$. Therefore, Eq. (119) can hold only if $f_{33}=0$.

We conclude:

$$
\frac{\varrho}{T} \frac{\partial \Psi}{\partial \nabla^{(n)} \boldsymbol{\varepsilon}}=\frac{\partial \underline{\underline{k}}}{\partial \nabla^{(n-1)} \dot{\boldsymbol{\varepsilon}}}
$$

The left-hand side of Eq. (120) depends on $\left(T, \boldsymbol{\varepsilon}, \nabla \boldsymbol{\xi}, \ldots, \nabla^{(n)} \boldsymbol{\varepsilon}\right)$, whereas the right-hand side depends on $\left(T, \dot{\boldsymbol{\varepsilon}}, \dot{\nabla} \boldsymbol{\varepsilon}, \ldots, \nabla^{(n-1)} \dot{\boldsymbol{\varepsilon}}\right)$. The only common variable is the temperature $T$, and we conclude that both sides of Eq. (120) can solely be functions of temperature. On the other hand, $\frac{\varrho}{T} \frac{\partial \Psi}{\partial \nabla^{(n)} \boldsymbol{\varepsilon}}$, as well as $\frac{\partial \underline{k}}{\partial \nabla^{(n-1)} \dot{\boldsymbol{\varepsilon}}}$ are tensors of order $n+2$. An isotropic tensor function cannot be represented by a scalar variable $T$. It follows:

$$
\frac{\varrho}{T} \frac{\partial \Psi}{\partial \nabla^{(n)} \boldsymbol{\varepsilon}}=0, \quad \frac{\partial \underline{k}}{\partial \nabla^{(n-1) \dot{\boldsymbol{\varepsilon}}}}=0 .
$$

Analogously, the line above in Eq. (111) is exploited and leads to the conclusions 


$$
\frac{\varrho}{T} \frac{\partial \Psi}{\partial \nabla^{(n-1)} \underline{\boldsymbol{\varepsilon}}}=0, \quad \frac{\partial \underline{k}}{\partial \nabla^{(n-2)} \dot{\boldsymbol{\varepsilon}}}=0 .
$$

The subsequent exploitation of the next lines up to the second but last one leads to the conclusions that

$$
\frac{\partial \Psi}{\partial \nabla \nabla \boldsymbol{\varepsilon}}=0, \quad \frac{\partial \Psi}{\partial \nabla \nabla \nabla \boldsymbol{\varepsilon}}=0, \ldots, \frac{\partial \Psi}{\partial \nabla^{(n-1)} \boldsymbol{\varepsilon}}=0, \quad \frac{\partial \Psi}{\partial \nabla^{(n)} \boldsymbol{\varepsilon}}=0
$$

and

$$
\frac{\partial \underline{k}}{\partial \nabla \dot{\boldsymbol{\varepsilon}}}=0, \quad \frac{\partial \underline{k}}{\partial \nabla \nabla \dot{\boldsymbol{\varepsilon}}}=0, \ldots, \frac{\partial \underline{k}}{\partial \nabla^{(n-2) \dot{\boldsymbol{\varepsilon}}}}=0, \quad \frac{\partial \underline{k}}{\partial \nabla^{(n-1) \dot{\boldsymbol{\varepsilon}}}}=0
$$

\subsection{Summary}

With the chosen set of variables equation (101) in the domain of the constitutive functions and including the power of the hyperstress $\underset{\underline{S}}{\boldsymbol{N}}$ in the balance of energy, we found from the exploitation of the second law of thermodynamics that the extra entropy flux $\underline{k}$ and the free energy density $\Psi$ depend solely on the following sets of variables:

$$
\begin{aligned}
& \underline{k}(T, \dot{\boldsymbol{\varepsilon}}), \\
& \Psi(T, \boldsymbol{\varepsilon}, \nabla \boldsymbol{\varepsilon}) .
\end{aligned}
$$

The free energy density does not depend on higher order strain gradients, but only on the strain gradients contributing to the internal power.

Remark: If we had set the hyperstress $\underset{\underline{S}}{\boldsymbol{S}}$ equal to zero, we could proceed with the evaluation of the second but last line of the entropy production Eq. (111). This leads to the conclusion that the extra entropy flux does not depend on $\dot{\xi}$, but can only depend on temperature.

In an isotropic material there exists no vector $\underline{k}$ depending on a scalar and a second-order symmetric tensor alone [44]. For isotropic materials, it can be concluded from Eq. (125) that the extra entropy flux vanishes for any order of gradients of strain included in the theory. These results are valid in general for any order of strain gradient included in the set of relevant variables in the beginning. In this sense, the inclusion of higher order strain gradients does not modify the results of the theory, at least concerning the constitutive function free energy density and the relation between entropy flux and heat flux. 


\subsection{Generalization to second-order time derivatives}

The set of variables in the domain of constitutive functions $\mathscr{Z}_{1}$ Eq. (101) considered here is general with respect to the order of space derivatives $n$ taken into account, but time derivatives are restricted to first-order ones. If secondorder time derivatives are included in the state space, i.e., we consider the domain of constitutive functions

$$
\mathscr{Z}_{2}=\left\{T, \nabla T, \boldsymbol{\varepsilon}, \nabla \underline{\boldsymbol{\varepsilon}}, \ldots, \nabla^{(n)} \boldsymbol{\varepsilon}, \dot{\boldsymbol{\varepsilon}}, \dot{\nabla}_{\boldsymbol{\varepsilon}}, \ldots, \nabla^{(n)} \dot{\boldsymbol{\varepsilon}}, \ddot{\boldsymbol{\varepsilon}}, \nabla \ddot{\boldsymbol{\varepsilon}}, \ldots, \nabla^{(n)} \ddot{\boldsymbol{\varepsilon}}\right\}
$$

the conclusions on the extra entropy flux and the free energy density are somewhat weaker:

1. The exploitation of the Liu equations (3) in that case imposes that the extra entropy flux does not depend on the highest space derivatives:

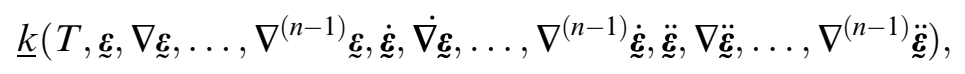

and the free energy density does not depend on the second-order time derivatives:

$$
\Psi\left(T, \boldsymbol{\varepsilon}, \nabla_{\boldsymbol{\varepsilon}}, \ldots, \nabla^{(n)} \boldsymbol{\xi}, \dot{\boldsymbol{\varepsilon}}, \dot{\nabla} \boldsymbol{\xi}, \ldots, \nabla^{(n)} \dot{\boldsymbol{\varepsilon}}\right) .
$$

2. The consecutive exploitation of the entropy production analogously to the previous examples leads to the conclusions:

(a) The extra entropy flux depends solely on time derivatives and on temperature:

$$
\underline{k}\left(T, \dot{\boldsymbol{\varepsilon}}, \nabla \dot{\boldsymbol{\varepsilon}}, \ldots, \nabla^{(n-1)} \dot{\boldsymbol{\varepsilon}}, \ddot{\boldsymbol{\varepsilon}}, \nabla \ddot{\boldsymbol{\varepsilon}}, \ldots, \nabla^{(n-1)} \ddot{\boldsymbol{\varepsilon}}\right) .
$$

(b) The dependence of $\underline{k}$ on $\nabla^{(n-1)} \ddot{\ddot{\varepsilon}}$ and of $\Psi$ on $\nabla^{(n)} \dot{\boldsymbol{\varepsilon}}$ is linear:

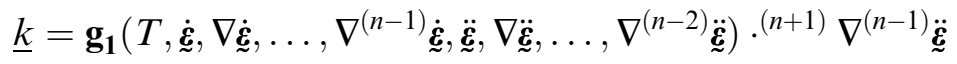

$$
\begin{aligned}
& +\underline{g_{2}}\left(T, \dot{\boldsymbol{\varepsilon}}, \nabla \dot{\boldsymbol{\varepsilon}}, \ldots, \nabla^{(n-1)} \dot{\boldsymbol{\varepsilon}}, \ddot{\boldsymbol{\varepsilon}}, \nabla \ddot{\boldsymbol{\varepsilon}}, \ldots, \nabla^{(n-2)} \ddot{\boldsymbol{\varepsilon}}\right)
\end{aligned}
$$

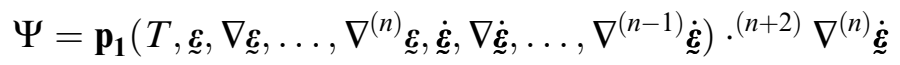

$$
\begin{aligned}
& +p_{2}\left(T, \boldsymbol{\varepsilon}, \nabla \boldsymbol{\varepsilon}, \ldots, \nabla^{(n)} \boldsymbol{\varepsilon}, \dot{\boldsymbol{\varepsilon}}, \nabla \dot{\boldsymbol{\varepsilon}}, \ldots, \nabla^{(n-1)} \dot{\dot{\boldsymbol{\varepsilon}}}\right),
\end{aligned}
$$

where $\underline{g}_{2}$ is a vector valued function, and $p_{2}$ is a scalar valued constitutive function. $\mathbf{g}_{\mathbf{1}}$ and $\mathbf{p}_{\mathbf{1}}$ are constitutive functions of tensor order $n+2$.

An interesting example is the case of gradients up to order $n=1$, i.e., constitutive functions depending on the variables $(T, \nabla T, \boldsymbol{\xi}, \nabla \boldsymbol{\varepsilon}, \dot{\boldsymbol{\varepsilon}}, \dot{\nabla} \boldsymbol{\varepsilon}, \ddot{\boldsymbol{\varepsilon}}, \nabla \ddot{\boldsymbol{\varepsilon}})$. The exploitation of the Liu equations leads to the conclusion that the extra entropy flux does not depend on gradients:

$$
\underline{k}(T, \boldsymbol{\varepsilon}, \dot{\boldsymbol{\varepsilon}}, \ddot{\boldsymbol{\varepsilon}}) .
$$


The exploitation of the entropy production shows that $k$ depends only on time derivatives of the strain, and the strain itself. The dependence on the second-order time derivative is linear:

$$
\underline{k}=\underline{\boldsymbol{k}}_{1}(T, \underline{\boldsymbol{\varepsilon}}, \dot{\boldsymbol{\varepsilon}}): \ddot{\ddot{\boldsymbol{\varepsilon}}}+\underline{\boldsymbol{k}}_{2}(T, \boldsymbol{\varepsilon}, \dot{\boldsymbol{\varepsilon}}) .
$$

In isotropic materials, there exists no third-order tensor $\underline{\underline{k}}_{1}$ and no vector $\underline{\boldsymbol{k}}_{2}$ depending on a scalar and a symmetric second-order tensor alone [44]. For materials of this symmetry, it can be concluded that the extra entropy flux vanishes, even if the possible dependence on second-order time derivatives is included at the beginning.

\section{Conclusions}

The restrictions on constitutive functions obtained in the different sections of this paper are summarized in Table 1. It has been shown that the free energy density of a thermoelastoviscoplastic solid may depend explicitly on an additional variable, on the gradient of the additional variable, or on the temperature gradient only if additional balance equations are introduced. Such extra field equations may be postulated independently as exposed in Section 4 or originate from an extension of the power of internal generalized forces as proposed in Section 3. As soon as such additional partial differential equations are introduced, the variable $\alpha$ ceases to be an "internal" variable and must be regarded as an actual degree of freedom, i.e., an observable and controllable quantity, even if the corresponding experimental effort may sometimes be considerable. In particular, extra boundary conditions are required compared to the classical framework. Their form was also derived from the systematic approach thus following [45]. Such an explicit dependence of the free energy on gradient variables is necessary to model size-dependent thermomechanical properties. Examples of nonlinear constitutive equations can

Table 1 Thermomechanical settings for strain gradient materials with gradient of internal variables and of temperature.

\begin{tabular}{|c|c|c|c|c|}
\hline Section & $\begin{array}{l}\text { Additional } \\
\text { differential } \\
\text { equations }\end{array}$ & $\begin{array}{l}\text { Enriched } \\
\text { power of } \\
\text { internal forces }\end{array}$ & $\begin{array}{l}\text { Free energy } \\
\text { density } \Psi\end{array}$ & $\begin{array}{l}\text { Extra-entropy } \\
\text { flux } \underline{\boldsymbol{k}}\end{array}$ \\
\hline 2 & no & no & $\Psi(T, \boldsymbol{\varepsilon}, \nabla \boldsymbol{\varepsilon})$ & $\underline{\boldsymbol{k}}(T, \dot{\boldsymbol{\varepsilon}})$ \\
\hline 3 & yes & yes & $\Psi(T, \boldsymbol{\varepsilon}, \alpha, \nabla T, \nabla \boldsymbol{\varepsilon}, \nabla \alpha)$ & $\boldsymbol{k}(T, \dot{T}, \dot{\boldsymbol{\varepsilon}}, \dot{\alpha})$ \\
\hline 4 & yes & no & $\Psi(T, \boldsymbol{\varepsilon}, \alpha, \nabla T, \nabla \tilde{\varepsilon}, \nabla \alpha)$ & $\begin{array}{r}\boldsymbol{k}(T, \boldsymbol{\varepsilon}, \alpha, \nabla T, \nabla \boldsymbol{\varepsilon}, \nabla \alpha, \\
\quad \dot{T}, \dot{\boldsymbol{\varepsilon}}, \dot{\alpha}, \nabla \dot{T}, \nabla \dot{\boldsymbol{\varepsilon}}, \nabla \dot{\alpha})\end{array}$ \\
\hline
\end{tabular}

The first column indicates whether additional differential equations are introduced. The second column indicates whether an extension of the power of internal forces is introduced or not, with respect to original expression for the second gradient theory of Mindlin [25, 26]. 
be found for instance in [18]. An alternative way, leading to a gradient dependent free energy density, can be found in [46-48], where in the exploitation of the dissipation inequality not only the evolution equations are taken into account, but also the gradients of evolution equations.

We have shown also that for isotropic materials, the second law of thermodynamics implies for a large class of state spaces (either including strain gradients of arbitrary order and first-order time derivatives of all these variables or including strain, strain gradient, and first-order as well as second-order time derivatives of these variables) that the extra entropy flux $\underline{k}$ vanishes, i.e., that the entropy flux equals the heat flux divided by temperature. In these cases, there is entropy transport only due to heat transport.

If materials have an internal structure that can change under the action of external fields, internal variables are introduced in order to deal with the more complicated material behavior [10]. Because these internal variables are unknown fields in addition to the classical wanted fields, equations of motion are needed for them. The equation of motion for an internal variable $\alpha$ (which can be a scalar, or a vector, or a tensor of arbitrary order) can be a relaxation equation of the form:

$$
\frac{d \alpha}{d t}=p_{\alpha}
$$

with a production $p_{\alpha}$, which is a state space function. For other additional variables, it can be an evolution-diffusion equation:

$$
\frac{d \alpha}{d t}+\nabla \cdot J_{\alpha}=P_{\alpha}
$$

with a production $P_{\alpha}$ and a flux of the additional variable $J_{\alpha}$. The case of a state space,

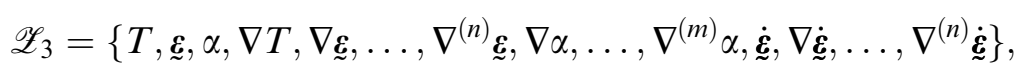

including in addition to the variables in Eq. (101) an internal variable of arbitrary tensor order and gradients of this internal variable, can be treated analogously to Sections 2.2 and 5.1, taking into account either the relaxation equation (135) for the internal variable or the evolution-diffusion equation (136). In the case of a relaxation equation for the internal variable, it can be shown that again the extra entropy flux vanishes, i.e., there is entropy transport only due to a heat flux, and the entropy flux is heat flux divided by temperature. In the other case of an evolution-diffusion equation for the additional variable, this conclusion on the entropy flux cannot be drawn. In this 
case, there is an extra entropy flux, which can be interpreted as the flux of entropy due the flow of the additional variable, i.e., flux of internal order.

\section{Acknowledgements}

Many valuable discussions with Prof. Muschik and Prof. Maugin and Dr. P. Ván are gratefully acknowledged. S. Forest thanks the Institut für Theoretische Physik of the Technical University of Berlin for financial support during his stay in Berlin in June/July 2003. C. Papenfuss thanks the Ecole des Mines de Paris/Centre des Matériaux for financial support during her stay in March/ April 2004.

\section{References}

[1] Germain, P., Nguyen, Q.S., Suquet, P., Continuum thermodynamics, J. Appl. Mechanics, 50 (1983), 1010-1020.

[2] Maugin, G.A., The Thermomechanics of Plasticity and Fracture, Chap. 7, Cambridge University Press, Cambridge, 1992.

[3] Lemaitre, J., Chaboche, J.-L., Mechanics of Solid Materials, University Press, Cambridge, UK, 1994.

[4] Verhas, J., Thermodynamics and Rheology, Akadémiai Kiadó, Budapest, 1997.

[5] Blenk, S., Ehrentraut, H., Muschik, W., Statistical foundation of macroscopic balances for liquid crystals in alignment tensor formulation, Physica A, 174 (1991), 119-138.

[6] Blenk, S., Ehrentraut, H., Muschik, W., Papenfuss, C., Mesoscopic orientation balances and macroscopic constitutive equations of liquid crystals, in: Proc. 7th Intl. Symp. on Continuum Models of Discrete Systems, volume 123-125 of Materials Science Forum, pp. 59-68, Paderborn, Germany, June 1992.

[7] Ehrentraut, H., Muschik, W., Papenfuss, C., Mesoscopically derived orientation dynamics of liquid crystals, J. Non-Equilib. Thermodyn., 22 (1997), 285-298.

[8] Blenk, S., Muschik, W., Mesoscopic concepts for constitutive equations of nematic liquid crystals in alignment tensor formulation, ZAMM, 73 (1993), T331T333.

[9] Maugin, G.A., Drouot, R., Internal variables and the thermodynamics of macromolecule solutions, Int. J. Eng. Sci., 21 (1983), 705-724.

[10] Maugin, G.A., Muschik, W., Thermodynamics with internal variables, J. NonEquilib. Thermodyn., 19 (1994), 217-249.

[11] Maugin, G.A., Muschik, W., Thermodynamics with internal variables, part ii. Applications, J. Non-Equilib. Thermodyn., 19 (1994), 250-289.

[12] Rice, J.R., Inelastic constitutive relations for solids: an internal-variable theory and its application to metal plasticity, J. Mech. Phys. Solids, 19 (1971), 433-455.

[13] Muschik, W., Internal variables in non-equilibrium thermodynamics, J. NonEquilib. Thermodyn., 15 (1990), 127-137.

[14] Ciancio, V., On the generalized Debye equation of media with dielectric relaxation phenomena described by vectorial internal thermodynamic variables, J. Non-Equilib. Thermodyn., 14 (1989), 239-250. 
[15] Ván, P., Internal thermodynamic variables and failure of microcracked materials, J. Non-Equilib. Thermodyn., 26 (2001), 167-189.

[16] Coleman, B.D., Gurtin, M.E., Thermodynamics with internal state variables, J. Chem. Phys., 47 (1967), 597-613.

[17] Mühlhaus, H.B., Continuum Models for Materials with Microstructure, Wiley, New York, 1995.

[18] Forest, S., Sievert, R., Elastoviscoplastic constitutive frameworks for generalized continua, Acta Mechanica, 160 (2003), 71-111.

[19] Ván, P., Weakly nonlocal irreversible thermodynambics, Annalen der Physik (Leipzig), 12 (2003), 142-169.

[20] Ván, P., Weakly nonlocal continuum theories of granular media: restrictions from the second law, Int. J. Solids Structures, 41 (2004), 5921-5927.

[21] Aifantis, E.C., The physics of plastic deformation, Int. J. Plasticity, 3 (1987), 211-248.

[22] Busso, E.B., Meissonnier, F.T., O’Dowd, N.P., Gradient-dependent deformation of two-phase single crystals, J. Mech. Phys. Solids, 48 (2000), 2333 2361.

[23] Acharya, A., Beaudoin, A.J., Grain size effects in viscoplastic polycrystals at moderate strains, J. Mech. Phys. Solids, 48 (2000), 2213-2230.

[24] Niordson, C.F., Hutchinson, J.W., On lower order strain gradient plasticity theories, Eur. J. Mech. A/Solids, 22 (2003), 771-778.

[25] Mindlin, R.D., Eshel, N.N., On first strain gradient theories in linear elasticity, Int. J. Solids Structures, 4 (1968), 109-124.

[26] Germain, P., La méthode des puissances virtuelles en mécanique des milieux continus, première partie: théorie du second gradient, J. Mécanique, 12 (1973), 235-274.

[27] Dillon, O.W., Kratochvíl, J., A strain gradient theory of plasticity, Int. J. Solids Structures, 6 (1970), 1513-1533.

[28] Dunn, J.E., Serrin, J., On the thermomechanics of interstitial working, Arch. Rat. Mech. Anal., 88 (1985), 96-133.

[29] Frémond, M., Nedjar, B., Damage, gradient of damage and principle of virtual power, Int. J. Solids Structures, 33 (1996), 1083-1103.

[30] Cardona, J.-M., Forest, S., Sievert, R., Towards a theory of second grade thermoelasticity, Extracta Mathematicae, 14 (1999), 127-140.

[31] Forest, S., Cardona, J.M., Sievert, R., Thermoelasticity of second-grade media, in: Continuum Thermomechanics, The Art and Science of Modelling Material Behaviour, Paul Germain's Anniversary Volume, Eds. G.A. Maugin, R. Drouot, F. Sidoroff, pp. 163-176, Kluwer Academic, Dordrecht, 2000.

[32] Svendsen, B., Continuum thermodynamic models for crystal plasticity including the effects of geometrically-necessary dislocations, J. Mech. Phys. Solids, 50 (2002), 1297-1329.

[33] Forest, S., Sievert, R., Aifantis, E.C., Strain gradient crystal plasticity: thermomechanical formulations and applications, J. Mech. Behavior Materials, 13 (2002), 219-232.

[34] Maugin, G.A., Internal variables and dissipative structures, J. Non-Equilib. Thermodyn., 15 (1990), 173-192.

[35] Lebon, G., Rubi, J.M., A generalized theory of thermoviscous fluids, J. NonEquilib. Thermodyn., 5 (1980), 285-300.

[36] Liu, I.S., Method of Lagrange multipliers for exploitation of the entropy principle, Arch. Rat. Mech. Anal., 46 (1972), 131-148. 
[37] Colemann, B.D., Noll, W., The thermodynamics of elastic materials with heat conduction and viscosity, Arch. Rat. Mech. Anal., 13 (1963), 167-168.

[38] Muschik, W., An amendment to the second law of thermodynamics, J. NonEquilib. Thermodyn., 21 (1996), 175-192.

[39] Liu, I.S., Müller, I., Extended thermodynamics of classical and degenerate gases, Arch. Rat. Mech. Anal., 83 (1983), 285.

[40] Muschik, W., Fundamental remarks on evaluating dissipation inequalities, in: Lecture Notes in Physics 199, Eds. J. Casas-Vazquez, D. Jou, G. Lebon, pp. 388-397, Springer, Berlin, Heidelberg, 1984.

[41] Ehrentraut, H., Muschik, W., Papenfuss, C., Concepts of Continuum Thermodynamics - 5 Lectures on Fundamentals, Methods and Examples, Sekcja Poligrafi Politechniki Swietokrzyskiej, Kielce, 1997.

[42] Liu, I.S., Continuum Mechanics, Springer, Berlin, Heidelberg, 2002.

[43] Müller, I., Grundzüge der Thermodynamik, Springer, Berlin, Heidelberg, 1994.

[44] Smith, G.F., On isotropic integrity bases, Arch. Rat. Mech. Anal., 17 (1964), 282-292.

[45] Maugin, G.A., The method of virtual power in continuum mechanics: application to coupled fields, Acta Mech., 35 (1980), 1-70.

[46] Ván, P., Exploiting the Second Law in weakly nonlocal continuum physics, Periodica Politechnica, Ser. Mechanical Engineering, 49 (2005), 79-94.

[47] Ciancio, V., Cimmelli, V.A., Ván, P., Balance laws for higher order fluxes in non-equilibrium thermodynamics, cond-mat/0407530, 2004.

[48] Cimmelli, V.A., Ván, P., The effects of nonlocality on the evolution of higher order fluxes in non-equilibrium thermodynamics, cond-mat/0409254, 2004.

Paper received: 2005-08-21

Paper accepted: 2006-01-19 
Bereitgestellt von | Technische Universität Berlin 